\title{
COMBINATORIAL HOMOTOPY. I
}

\author{
J. H. C. WHITEHEAD
}

1. Introduction. This is the first of a series of papers, whose aim is to clarify the theory of "nuclei" and " $n$-groups" and its relation to Reidemeister's ${ }^{1}$ Überlagerungen. Here we give a new definition of " $n$-groups," or $n$-types as we now propose to call them. This is stated in terms of $(n-1)$-homotopy types, which were introduced by $\mathrm{R} . \mathrm{H}$. Fox. ${ }^{2}$ In a later paper we shall show that this is equivalent to the definition in terms of elementary transformations, which was given in [1]. The series of $n$-types $(n=1,2, \cdots)$ is a hierarchy of homotopy, and a fortiori of topological invariants. That is to say, if two complexes, ${ }^{3} K, L$, are of the same $n$-type, then they are of the same $m$-type for any $m<n$, where $n \leqq \infty$ and the $\infty$-type means the homotopy type. If $\operatorname{dim} K, \operatorname{dim} L \leqq n$ then $K, L$ are of the same homotopy type if they are of the same $(n+1)$-type. Two complexes are of the same 2-type if, and only if, their fundamental groups are isomorphic. Moreover any (discrete) group is isomorphic to the fundamental group of a suitably constructed complex. Therefore the classification of complexes according to their 2-types is equivalent to the classification of groups by the relation of isomorphism. Thus the $n$-type $(n>2)$ is a natural generalization of a geometrical equivalent of an abstract group. ${ }^{4}$

Following up this idea we look for a purely algebraic equivalent of an $n$-type when $n>2$. An important requirement for such an algebraic system is "realizability," in two senses. In the first instance this means that there is a complex which is in the appropriate relation to a given one of these algebraic systems, just as there is a complex whose fundamental group is isomorphic to a given group. The second kind, whose importance is underlined by theorems in $[5 ; 6]$ and in this paper, is the "realizability" of homomorphisms, chain mappings, etc., by maps of the corresponding complexes. Thus realizability means that the algebraic representation is not subject to conditions which can only be expressed geometrically.

An address delivered before the Princeton Meeting of the Society on November 2, 1946, by invitation of the Committee to Select Hour Speakers for Eastern Sectional Meetings; received by the editors July 19, 1948.

${ }^{1}$ See $[1],[3]$ and $[8$, p. 177$]$. Numbers in brackets refer to the references cited at the end of the paper.

${ }^{2}$ See $[9$, p. 343$]$ and $[10$, p. 49$]$.

${ }^{3}$ I.e., CW-complexes, as defined in $\$ 5$ below.

${ }^{4}$ I.e., the class of groups which are isomorphic to a given group. 
What has been achieved so far is a purely algebraic description of the homotopy type of any 3-dimensional complex (see [3]) and of any finite, simply-connected, 4-dimensional complex. An account of the former will be given in Paper II of this series and of the latter in [5]. This and Theorem 6 below lead to an algebraic description of the 3-type of any complex and of the 4-type of any simply-connected, finite complex. The theorem on the realizability of chain-mappings, which applies to the 3-dimensional complexes, will be generalized to what we call $J_{m}$-complexes, which are defined in $\$ 3$ below.

The algebraic apparatus used in [5] and in Paper II is inadequate for the classification of homotopy classes of maps of such complexes. Each homotopy class of maps induces a unique equivalence class of algebraic maps. Moreover each algebraic map, of the appropriate algebraic type, has a geometrical realization. But in general the homotopy class of the latter is not unique. For example in the case of a simply-connected, 3-dimensional complex our algebraic system simply consists of the ordinary chain groups and all maps of a 3-sphere in a 2-sphere lead to the same equivalence class of chain mappings. Thus our results are complementary to much of the recent work on the algebraic classification of mappings (e.g. [11]).

In this presentation we abandon simplicial complexes in favor of cell complexes. This first part consists of geometrical preliminaries, including some elementary propositions concerning what we call closure finite complexes with weak topology, abbreviated to CW-complexes, which are defined in $\$ 5$ below. There are two main reasons why we do not confine ourselves to finite complexes. The first is that we want to include such simple spaces as open manifolds or, more generally, infinite but locally finite complexes. The second is that we have a great deal to do with covering complexes. We do not restrict ourselves to locally finite complexes because this restriction would be troublesome when considering "mapping cylinders" of infinite complexes. Also we want to allow for such operations as shrinking an infinite subcomplex (e.g., a tree containing all the 0-cells) into a point.

We recall from [6] that a space, $P$, dominates a space, $X$, if, and only if, there are maps, $\lambda: X \rightarrow P, \lambda^{\prime}: P \rightarrow X$, such that $\lambda^{\prime} \lambda \simeq 1$. We show that the theorems in [6] can be extended to non-compact spaces, which are dominated by $\mathrm{CW}$-complexes. To do this we give a new and possibly ${ }^{5}$ more restrictive definition of $n$-homotopy. According to Fox, maps $f, g: X \rightarrow Y$, of any space $X$ in a space $Y$, are $n$-homotopic, written $f \simeq_{n} g$, if, and only if, $f \phi \simeq g \phi$, for every map,

' See $\$ 7$ below. 
$\phi: K^{n} \rightarrow X$, of every finite simplicial complex, $K^{n}$, of at most $n$ dimensions. We alter this by defining $f \simeq_{n} g$ if, and only if, $f \phi \simeq_{g \phi}$ for every map, $\phi: K^{n} \rightarrow X$, of every CW-complex, $K^{n}$, of at most $n$ dimensions. As in [6] we describe a map, $f: X \rightarrow Y$, as an $n$-homotopy equivalence if, and only if, it has an $n$-homotopy inverse, meaning a map, $g: Y \rightarrow X$, such that $g f \simeq_{n} 1, f g \simeq_{n} 1$. A homotopy equivalence is similarly defined in terms of ordinary homotopy. We shall use the symbol

$$
X \equiv_{n} Y
$$

to mean that $X$ and $Y$ are of the same $n$-homotopy type (i.e. there is an $n$-homotopy equivalence $f: X \rightarrow Y$ ) and $X \equiv Y$ will mean that $X$ and $Y$ are of the same homotopy type.

Let $\alpha$ be the class of all connected spaces, each of which is dominated by some CW-complex. ${ }^{6}$ Let $\alpha_{0} \subset \alpha$ be the subclass consisting of spaces which are locally simply-connected in the weak sense. That is to say, if $X \in \alpha_{0}$ there is a basic set of neighborhoods in $X$, such that any closed curve in one of these neighborhoods is contractible to a point in $X$. The universal covering space, $\tilde{X}$, of such a space has the usual properties. If $X$ is dominated by a CW-complex of finite dimensionality we shall use $\Delta X$ to denote the minimum dimensionality of all $\mathrm{CW}$-complexes which dominate $X$. If none of the CW-complexes, which dominate $X$, has finite dimensionality we write $\Delta X=\infty$. We restate the theorems in [6], which now refer to spaces $X, Y \subset \alpha$ and to the new definition of $n$-homotopy. Let $f_{n}: \pi_{n}(X) \rightarrow \pi_{n}(Y)$ be the homomorphism induced by a map $f: X \rightarrow Y(n=1,2, \cdots)$ and let $N=\max (\Delta X, \Delta Y) \leqq \infty$.

THEOREM 1. The map $f: X \rightarrow Y$ is a homotopy equivalence if, and only if, $f_{n}: \pi_{n}(X) \rightarrow \pi_{n}(Y)$ is an isomorphism onto for every $n$ such that $1 \leqq n<N+1$.

TheOREM 2. The map $f: X \rightarrow Y$ is an $(N-1)$-homotopy equivalence if, and only if, $f_{n}$ is an isomorphism onto for every $n$ such that $1 \leqq n<N$.

Let $X, Y \subset \alpha_{0}$ and let $\tilde{X}, \tilde{Y}$ be the universal covering spaces of $X$, $Y$, with base points $x_{0} \in X$ and $y_{0}=f x_{0} \in Y$. As explained in [6], the map $f: X \rightarrow Y$ induces homomorphisms $H_{n}(\tilde{X}) \rightarrow H_{n}(\tilde{Y})$, where $H_{n}$ indicates the $n$th homology group, defined in terms of singular cycles. The argument given in [6] shows that $H_{n}(\tilde{X})=0, H_{n}(\tilde{Y})=0$ if $n>N$.

${ }^{6}$ A space in $\alpha$ must satisfy the usual union and intersection axioms for closed and open sets but need not satisfy any separation axioms. For example, a single point need not be a closed subset. It follows from $(M)$ in $\$ 5$ below that any connected $\mathrm{CW}$-complex is arcwise connected. Hence it is easily proved that every space in $\alpha$ is arcwise connected. 
TheOREM 3. The map $f: X \rightarrow Y$ is a homotopy equivalence if, and only if, each of the induced homomorphisms $f_{1}: \pi_{1}(X) \rightarrow \pi_{1}(Y)$ and $H_{n}(\tilde{X})$ $\rightarrow H_{n}(\tilde{Y})$ is an isomorphism onto.

Let $N<\infty$.

THEOREM 4. The map $f: X \rightarrow Y$ is an $(N-1)$-homotopy equivalence if

(a) each of the induced homomorphisms $f_{1}: \pi_{1}(X) \rightarrow \pi_{1}(Y), H_{n}(\tilde{X})$ $\rightarrow H_{n}(\tilde{Y})$ is an isomorphism onto, for $n=0, \cdots, N-2$, and also

(b) the induced homomorphism $H_{N-1}(\tilde{X}) \rightarrow H_{N-1}(\tilde{Y})$ is onto and

(c) $f_{N-1}: \pi_{N-1}(X) \rightarrow \pi_{N-1}(Y)$ is an isomorphism into.

Conversely if $f: X \rightarrow Y$ is an $(N-1)$-homotopy equivalence, ${ }^{7}$ so is the lifted map, $\tilde{f}: \tilde{X} \rightarrow \tilde{Y}$, and $H_{n}(\tilde{X}) \rightarrow H_{n}(\tilde{Y})$ is an isomorphism onto for $n=0, \cdots, N-1$.

2. $n$-types. Let $K, L$ be $C W$-complexes. A map $f: K \rightarrow L$ is said to be cellular if, and only if, $f K^{n} C L^{n}$ for each $n \geqq 0$, where $K^{n}, L^{n}$ are the $n$ sections of $K, L$. A homotopy $f_{t}: K \rightarrow L$ is said to be cellular if, and only if, the maps $f_{0}, f_{1}$ are cellular and $f_{t} K^{n} C L^{n+1}$ for each $n \geqq 0$. According to (L), in $\$ 5$ below, any map $K \rightarrow L$ is homotopic to a cellular map and if $f_{0} \simeq f_{1}: K \rightarrow L$, where $f_{0}, f_{1}$ are cellular, then there is a cellular homotopy $f_{t}: K \rightarrow L$. Let $f_{0}, f_{1}: L \rightarrow X$ be maps of $L$ in any space $X$ and let $f_{0} \simeq_{n} f_{1}$. Then $f_{0} \phi \simeq f_{1} \phi$ for any map, $\phi: K^{n} \rightarrow L$, of any CW-complex, $K^{n}$, of at most $n$ dimensions. In particular $f_{0} \mid L^{n}$ $\simeq f_{1} \mid L^{n}$. But $\phi$ is homotopic to a cellular map, $\phi^{\prime}: K^{n} \rightarrow L$, and if $f_{0}\left|L^{n} \simeq f_{1}\right| L^{n}$ we have $f_{0} \phi \simeq f_{0} \phi^{\prime} \simeq f_{1} \phi^{\prime} \simeq f_{1} \phi$. Therefore $f_{0} \simeq_{n} f_{1}$ if, and only if, $f_{0}\left|L^{n} \simeq f_{1}\right| L^{n}$. If $\operatorname{dim} L \leqq n$ then $f_{0} \simeq_{n} f_{1}$ obviously means $f_{0} \simeq f_{1}$. If will be convenient to allow $n$ to take the value $\infty$ on the understanding that $L^{\infty}=L$ and that $f_{0} \simeq_{\infty} f_{1}$ and $K \equiv{ }_{\infty} L$ mean $f_{0} \simeq f_{1}$ and $K \equiv L$.

We shall say that $\mathrm{CW}$-complexes, $K, L$, are of the same $n$-type $(1 \leqq n \leqq \infty)$ if, and only if, $K^{n} \equiv_{n-1} L^{n}$. If $\operatorname{dim} K, \operatorname{dim} L \leqq n$, then $X^{n+1}=X^{n}=X(X=K$ or $L)$ and $K^{n+1} \equiv{ }_{n} L^{n+1}$ means $K \equiv L$.

Theorem 5. If $K$ and $L$ are of the same $n$-type $(2 \leqq n \leqq \infty)$ then they are of the same $m$-type for any $m<n$.

Let $f: K^{n} \rightarrow L^{n}$ be an $(n-1)$-homotopy equivalence and let $g: L^{n}$ $\rightarrow K^{n}$ be an $(n-1)$-homotopy inverse of $f$. We may assume that $f$ and $g$ are cellular maps and also that there are cellular homotopies, $\xi_{t}: K^{n-1} \rightarrow K^{n}, \eta_{t}: L^{n-1} \rightarrow L^{n}$, such that $\xi_{0}=g f\left|K^{n-1}, \xi_{1}=1, \eta_{0}=f g\right| L^{n-1}$,

${ }^{7}$ By taking $X$ to be a 2 -sphere and $Y$ to be a complex projective plane one sees that $f: X \rightarrow Y$ need not be an $(N-1)$-homotopy equivalence $(N=4)$ even though the induced homomorphisms $f_{1}$ and $H_{n}(\widetilde{X}) \rightarrow H_{n}(\widetilde{Y})$ are isomorphisms onto for $n=1, \cdots$, $N-1$. 
$\eta_{1}=1$, where 1 stands for both identical maps $K^{n-1} \rightarrow K^{n}, L^{n-1} \rightarrow L^{n}$. Let $f^{m}: K^{m} \rightarrow L^{m}, g^{m}: L^{m} \rightarrow K^{m}$ be the maps induced by $f, g$ (i.e. $f^{m} p=f p$, $g^{m} q=g q$, where $\left.p \in K^{m}, q \in L^{m}\right)$. Since $\xi_{t} K^{m-1} \subset K^{m}$ it follows that $g^{m} f^{m} \mid K^{m-1} \simeq 1$ (in $K^{m}$ ). Therefore $g^{m} f^{m} \simeq_{m-1} 1$. Similarly $f^{m} g^{m} \simeq_{m-1} 1$, whence $K^{m} \equiv_{m-1} L^{m}$ and the theorem is proved.

It follows from Theorem 5 that the $n$-type is an invariant of the homotopy type (i.e. is the same for two complexes of the same homotopy type). It is a fortiori a topological invariant. Since a CWcomplex is locally contractible, according to $(\mathrm{M})$ in $\$ 5$ below, each component is arcwise connected. Therefore two CW-complexes have the same 1-type if, and only if, they have the same cardinal number of components. It also follows from the results in $\$ 5$ below, and the argument used in the finite case, ${ }^{8}$ that connected CW-complexes, $K, L$, have the same 2-type if, and only if, $\pi_{1}(K) \approx \pi_{1}(L)$. We now assume, until $\S 4$, that any given complex is connected and that $n \geqq 2$ in statements concerning $n$-homotopy and $n$-types.

These ideas provide a generalization of a result due to Hopf, ${ }^{9}$ on the relation between the fundamental group and the second homology group of a polyhedron. For let $X$ be any space and let $G_{n}(X)$ be the residue group

$$
G_{n}(X)=H_{n}(X)-S_{n}(X),
$$

where $S_{n}(X) \subset H_{n}(X)$ is the sub-group whose elements are represented by spherical cycles. Let $f, g: X \rightarrow Y$ be maps of $X$ in a space $Y$. It may be proved that, ${ }^{10}$ if $f \simeq_{n-1} g$, then $f$ and $g$ induce the same homomorphism $G_{n}(X) \rightarrow G_{n}(Y)$. It follows that, if $X \equiv_{n-1} Y$, then $G_{n}(X) \approx G_{n}(Y)$. In case $X, Y$ are CW-complexes this also follows from:

THEOREM 6. If $K_{1}^{n} \equiv_{n-1} K_{2}^{n}$ then

$$
K_{1}^{n} \cup \Sigma_{1}^{n} \equiv K_{2}^{n} \cup \Sigma_{2}^{n}
$$

where $\Sigma_{i}^{n}(i=1,2)$ is a set of (disjoint) $n$-cells, whose closures are $n$ spheres, attached to $K_{i}^{n}$ at a single point, $\Sigma_{i}^{n}$ being a finite set of cells if $K_{1}^{n}, K_{2}^{n}$ are finite complexes.

This is Theorem 13 in $^{11}[1]$, restated in terms of CW-complexes

${ }^{8}$ See $[16$, pp. 217,213$]$, and $[18,3.1,3.2$ on p. 29$]$.

- See [17] and [18]. See also [1, Theorems 12 and 13, pp. 266, 269].

10 This is easily proved on the assumption that any homology class in $H_{n}(X)$ has a representative (singular) cycle, which is also a continuous cycle (i.e., the image in a map, $K^{n} \rightarrow X$, of a cycle carried by a finite, $n$-dimensional, simplicial complex $K^{n}$ ).

11 [1], p. 269 for finite and p. 324 for infinite complexes. 
and the new definition of the $n$-type. We shall give another proof in a later paper.

Another corollary of Theorem 6, and of Theorem 19 in [1] is:

CoROLlaRY 1. If $K_{1}^{n} \equiv_{n-1} K_{2}^{n}$ then $^{12}$

$$
\pi_{n}\left(K_{1}^{n}\right)+M_{1} \approx \pi_{n}\left(K_{2}^{n}\right)+M_{2},
$$

where + indicates direct summation and $M_{i}$ is a free $\Re_{i}$-module, $\Re_{i}$ being the group ring of $\pi_{1}\left(K_{i}\right)$ with integral coefficients.

Another obvious corollary of Theorem 6, which is also easy to prove directly, is:

COROLlARY 2. If $K_{1}^{n}$ and $K_{2}^{n}$ are of the same n-type, so are their universal covering complexes, $\tilde{K}_{1}^{n}$ and $\tilde{K}_{2}^{n}$.

Hence, using $G_{n}(X)$ to mean the same as before, with $X=\tilde{K}_{\imath}^{n}$, we have:

COROLlARY 3. If $K_{1}^{n} \equiv_{n-1} K_{2}^{n}$ then $G_{n}\left(\tilde{K}_{1}^{n}\right) \approx G_{n}\left(\tilde{K}_{2}^{n}\right)$.

3. $J_{m}$-complexes. Let $K$ be a (connected) CW-complex and let a 0 -cell, $e^{0}$, be taken as base point for all the groups $\pi_{n}\left(K^{n}, K^{n-1}\right)$, $\pi_{r}\left(K^{r}\right)(n \geqq 2, r \geqq 1)$. Let $\rho_{n}=\pi_{n}\left(K^{n}, K^{n-1}\right)$ and let $j_{n}: \pi_{n}\left(K^{n}\right) \rightarrow \rho_{n}$ be the homomorphism induced by the identical map $\left(K^{n}, e^{0}\right) \rightarrow\left(K^{n}\right.$, $\left.K^{n-1}\right)$. We shall describe $K$ as a $J_{m}$-complex if, and only if, $j_{n}$ is an isomorphism into $\rho_{n}$ for each $n=2, \cdots, m$. Obviously $K$ is a $J_{m^{-}}$ complex if, and only if, $K^{m}$ is a $J_{m}$-complex. We shall prove that the property of being a $J_{m}$-complex is an invariant of the $m$-type. That is to say, if $K^{m} \equiv_{m-1} L^{m}$ and if $K$ is a $J_{m}$-complex so is $L$.

Let $\beta_{n}: \rho_{n} \rightarrow \pi_{n-1}\left(K^{n-1}\right)(n \geqq 2)$ be the (homotopy) boundary homomorphism and let

$$
d_{n}=j_{n-1} \beta_{n}: \rho_{n} \rightarrow \rho_{n-1},
$$

where $\rho_{1}=\pi_{1}\left(K^{1}\right)$ and $j_{1}=1$. Then $d_{n} j_{n}=0$ and $d_{n} d_{n+1}=0$ since $\beta_{n} j_{n}=0$. Let $i_{n}: \pi_{n}\left(K^{n-1}\right) \rightarrow \pi_{n}\left(K^{n}\right)$ be the homomorphism induced by the identical map $K^{n-1} \rightarrow K^{n}$.

We now prove two theorems which are closely related to Theorem 1 in [25].

TheOREM 7. The homomorphism $j_{n}: \pi_{n}\left(K^{n}\right) \rightarrow \rho_{n}$ is into $d_{n}^{-1}(0)(n \geqq 2)$.

12 In each of Corollaries 1 and 3 the symbol $\approx$ means that there is an operator isomorphism (onto), which is defined in terms of the isomorphism, $\pi_{1}\left(K_{1}^{n}\right) \rightarrow \pi_{1}\left(K_{2}^{n}\right)$, induced by an $(n-1)$-homotopy equivalence $K_{1}^{n} \rightarrow K_{2}^{n}$. Compare Corollary 3 with Theorem 28.1 on p. 414 of [13]. 
It is:

(a) an isomorphism (into) if, and only if, $i_{n} \pi_{n}\left(K^{n-1}\right)=0$ and is

(b) onto $d_{n}^{-1}(0)$ if $i_{n-1} \pi_{n-1}\left(K^{n-2}\right)=0$.

The fact that $j_{n} \pi_{n}\left(K^{n}\right) \subset d_{n}^{-1}(0)$ follows from the relation $d_{n} j_{n}=0$. The assertion (a) follows at once from the exactness of the homotopy sequence

$$
\pi_{r}\left(K^{r-1}\right) \stackrel{i}{\rightarrow} \pi_{r}\left(K^{r}\right) \stackrel{j}{\rightarrow} \pi_{r}\left(K^{r}, K^{r-1}\right) \stackrel{\beta}{\rightarrow} \pi_{r-1}\left(K^{r-1}\right),
$$

with $r=n$. Let $a \in d_{n}^{-1}(0)$ be given $(n \geqq 2)$. Since $d_{n} a=j_{n-1} \beta_{n} a=0$ it follows from the exactness of (3.1) with $r=n-1$ and $\pi_{1}\left(K^{0}\right)=\pi_{0}\left(K^{0}\right)$ $=0$ in case $n=2$, that $\beta_{n} a \in i_{n-1} \pi_{n-1}\left(K^{n-2}\right)$. Therefore $\beta_{n} a=0$ if $i_{n-1} \pi_{n-1}\left(K^{n-2}\right)=0$ and it follows from the exactness of (3.1), with $r=n$, that $a \in j_{n} \pi_{n}\left(K^{n}\right)$. This completes the proof.

Since $\pi_{2}\left(K^{1}\right)=0$ it follows from Theorem 7 that any complex is a $J_{2}$-complex. Also $j_{2}$ is onto $d_{2}^{-1}(0)=\beta_{2}^{-1}(0)$ by the exactness of (3.1) with $r=2$. In general we have:

Corollary. $A$ complex, $K$, is a $J_{m}$-complex if, and only if, $i_{n} \pi_{n}\left(K^{n-1}\right)$ $=0$ for $n=2, \cdots, m$. In this case $j_{r}$ is onto $d_{r}^{-1}(0)$ for $r=2, \cdots, m+1$.

Let $\pi_{1}(K)=1$. Then the groups $\rho_{n}(n>2)$ and $\rho_{1}, \rho_{2}$ made Abelian may be taken as chain-groups. ${ }^{13}$ The homology group, $H_{n}(K)$, may be defined as the residue group

$$
H_{n}(K)=d_{n}^{-1}(0)-d_{n+1} \rho_{n+1}=d_{n}^{-1}(0)-j_{n} \beta_{n+1} \rho_{n+1} .
$$

It follows from (L), in $\$ 5$ below, that we may take

$$
\pi_{n}(K)=\pi_{n}\left(K^{n+1}\right)=\pi_{n}\left(K^{n}\right)-\beta_{n+1} \rho_{n+1} .
$$

Therefore $j_{n}$ determines a homomorphism $j_{n}^{*}: \pi_{n}(K) \rightarrow H_{n}(K)$.

TheOREM 8. If $\pi_{1}(K)=1$ and if $K$ is a $J_{m}$-complex, then $j_{n}^{*}: \pi_{n}(K)$ $\rightarrow H_{n}(K)$ is an isomorphism onto for $n=1, \cdots, m$ and $j_{m+1}^{*}$ is onto.

This is an immediate consequence of (3.2), (3.3) and Theorem 7 and its corollary.

We shall say that a space $Y, n$-dominates a space $X$ if, and only if, there is a map, $f: X \rightarrow Y$, which has a left $n$-homotopy inverse, meaning a map $g: Y \rightarrow X$ such that $g f \simeq_{n} 1$.

Theorem 9. If a CW-complex, $K$, is $(m-1)$-dominated by a $J_{m}$ complex, $L$, then $K$ is a $J_{m}$-complex.

${ }^{13} \mathrm{Cf}$. [6], [5]. For the definition of $H_{n}(K)$ in terms of the relative homology groups $H_{n}\left(K^{n}, K^{n-1}\right)$, treated as chain groups, see [14]. 
Let $f: K \rightarrow L$ and $g: L \rightarrow K$ be cellular maps such that $g f \simeq_{m-1} 1$. Since $f, g$ are cellular they induce homomorphisms

$$
\begin{aligned}
& \phi_{n}: i_{n} \pi_{n}\left(K^{n-1}\right) \rightarrow i_{n} \pi_{n}\left(L^{n-1}\right), \\
& \psi_{n}: i_{n} \pi_{n}\left(L^{n-1}\right) \rightarrow i_{n} \pi_{n}\left(K^{n-1}\right) .
\end{aligned}
$$

Let $\xi_{t}: K^{m-1} \rightarrow K^{m}$ be a cellular homotopy such that $\xi_{0}=g f \mid K^{m-1}, \xi_{1}=1$. Let $2 \leqq n \leqq m$. Then $\xi_{t} K^{n-1} \subset K^{n}$ and it follows that $\psi_{n} \phi_{n}=\alpha_{n}$, where $\alpha_{n}$ is an automorphism of $i_{n} \pi_{n}\left(K^{n-1}\right)$. Therefore $\psi_{n}$ is onto. But $i_{n} \pi_{n}\left(L^{n-1}\right)=0$, by the corollary to Theorem 7 . Therefore $i_{n} \pi_{n}\left(K^{n-1}\right)$ $=0$, whence $K$ is a $J_{m}$-complex and Theorem 9 is proved.

If $K^{m} \equiv_{m-1} L^{m}$ then $K^{m}$ and $L^{m}(m-1)$-dominate each other. Therefore we have the corollary:

COROLLARY. The property of being a $J_{m}$-complex is an invariant of the m-type.

Let $K$ be a CW-complex such that $\pi_{n}(K)=0$ for $n=1, \cdots, m-1$. Then it follows from Theorem 2 that the map $K^{m} \rightarrow L^{0}$ is an $(m-1)$ homotopy equivalence, where $L^{0}$ is a single point. Since $L^{0}$ is (obviously) a $J_{m}$-complex it follows from Theorem 9 that $K^{m}$ and hence $K$ is a $J_{m}$-complex. Therefore Theorem 8 includes Hurewicz's result ${ }^{14}$ that $\pi_{m}(K) \approx H_{m}(K)$ if $\pi_{n}(K)=0$ for $n=1, \cdots, m-1$. The following example of a simply connected $J_{4}$-complex, $K$, with $\pi_{3}(K) \neq 0$, shows that Theorem 8 is more general than Hurewicz's theorem, restricted to $\mathrm{CW}$-complexes. Let $K=e^{0} \cup e^{3} \cup e^{4}$, where $e^{0}$ is a 0 -cell, $e^{3}$ is a 3 -cell whose closure is a 3 -sphere, $S^{3}=e^{0} \cup e^{3}$, and $e^{4}$ is attached ${ }^{15}$ to $S^{3}$ by a map, $f: \partial E^{4} \rightarrow S^{3}$, of degree $(2 r+1)(r>0)$. Then $\pi_{3}(K)$ is cyclic of order $2 r+1$. If $1 \leqq n \leqq 3$ then $K^{n-1}=K^{0}$, whence $\pi_{n}\left(K^{n-1}\right)=0$. Let $g: S^{4} \rightarrow \partial E^{4}$ be an essential map. Then $f g: S^{4} \rightarrow S^{3}$ is essential [4, Theorem 9, p. 268] and hence represents the nonzero element of $\pi_{4}\left(S^{3}\right)$. Therefore [2, Lemma 4, p. 418] $i_{4} \pi_{4}\left(K^{3}\right)=0$, whence $K$ is a $J_{4}$-complex.

According to (M) and (N) in $\S 5$ below, a CW-complex, $K$, is locally contractible and its universal covering complex, $\tilde{K}$, is also a CWcomplex.

TheOREm 10. $K$ is a $J_{m}$-complex if, and only if, $\tilde{K}$ is a $J_{m}$-complex.

This is an obvious consequence of the corollary to Theorem 7 .

${ }^{14}[15$, p. 522$]$. See also $[19$, p. 314$]$. Hurewicz's theorem applies to a larger class of spaces than $\mathrm{CW}$-complexes.

${ }^{16}$ See $\$ 8$ below. We use $E^{n}$ to denote an $n$-element (i.e. a homeomorph of an $n$-simplex) and $\partial E^{n}$ to denote its boundary. By a simplex we mean a simplex with boundary. 
Let $\tilde{K}$ be the universal covering complex of a CW-complex $K$. Since $\pi_{1}(\tilde{K})=1, \pi_{n}(\tilde{K}) \approx \pi_{n}(K)$ if $n>1$ and since $L$ is a $J_{m}$-complex if $\pi_{r}(L)=0$ for $r=1, \cdots, m-1$ it follows from Theorem 10 that $K$ is a $J_{m}$-complex if $\pi_{n}(K)=0$ for $n=2, \cdots, m-1$. In particular $K$ is a $J_{m}$-complex if its universal covering space is an $m$-sphere or any Euclidean space.

In Paper II we shall consider "Reidemeister" systems of chain groups, $C(K), C(L)$ and chain mappings $C(K) \rightarrow C(L)$, where $K, L$, are CW-complexes. We shall prove that, if $L$ is a $J_{m}$-complex and if $\operatorname{dim} K \leqq m+1$, then any chain mapping, $C(K) \rightarrow C(L)$, can be realized by some (cellular) map, $K \rightarrow L$. Hence it follows from Theorem 3 that $K \equiv L$ if there is a chain equivalence $C(K) \rightarrow C(L)$. If, in addition [5, Lemma 4], $K$ and $L$ are finite, simply-connected complexes, then $K \equiv L$ if $H_{n}(K) \approx H_{n}(L)$ for all values of $n$.

4. Cell complexes. ${ }^{16}$ By a cell complex, $K$, or simply a complex, we mean a Hausdorff space, which is the union of disjoint (open) cells, to be denoted by $e, e^{n}, e_{i}^{n}$, etc., subject to the following condition. The closure, $\bar{e}^{n}$, of each $n$-cell, $e^{n} \in K$, shall be the image of a fixed $n$-simplex, $\sigma^{n}$, in a map, $f: \sigma^{n} \rightarrow \bar{e}^{n}$, such that

(4.1) (a) $f \mid \sigma^{n}-\partial \sigma^{n}$ is a homeomorphism onto $e^{n}$,

(b) $\partial e^{n} C K^{n-1}$, where $\partial e^{n}=f \partial \sigma^{n}=\bar{e}^{n}-e^{n}$ and $K^{n-1}$ is the $(n-1)$ section ${ }^{17}$ of $K$, consisting of all the cells whose dimensionalities do not exceed $n-1$.

Such a map will be called a characteristic map for the cell $e^{n}$. If $f: \sigma^{n} \rightarrow \bar{e}^{n}$ is a characteristic map for $e^{n}$, so obviously is $f h: \sigma^{n} \rightarrow \bar{e}^{n}$, where $h:\left(\sigma^{n}, \partial \sigma^{n}\right) \rightarrow\left(\sigma^{n}, \partial \sigma^{n}\right)$ is any map such that $h \mid \sigma^{n}-\partial \sigma^{n}$ is a homeomorphism of $\sigma^{n}-\partial \sigma^{n}$ onto itself. No restriction other than $\partial e^{n} \subset K^{n-1}$ is placed on $f \mid \partial \sigma^{n}$. Therefore $\bar{e}^{n}$ need not coincide, as a point set, with a subcomplex of $K$. Since $K$, and hence $\bar{e}^{n}$, is a Hausdorff space and since $\sigma^{n}$ is compact it follows that $\bar{e}^{n}$ has the identification topology determined ${ }^{18}$ by $f$. A complex is defined as a topological space with a certain cell structure. Therefore we shall not need a separate letter to denote a complex and the space on which it lies. ${ }^{19}$ Notice that, in the absence of further restrictions, any (Hausdorff) space may be re-

${ }^{16}$ The use of these complexes was suggested in $[3$, p. 1235]. They are now called cell complexes, rather than membrane complexes, in conformity with [14].

${ }^{17} K^{n}$ is defined for every value of $n$. If there are no $m$-cells in $K$ for $m>n$ then $K^{n}=K$.

${ }^{18}$ I.e., $Y \subset \bar{e}^{n}$ is closed if, and only if, $f^{-1} Y$ is closed. In other words the closed sets in $\bar{e}^{n}$ are precisely the sets $f X$ for every closed set, $X \subset \sigma^{n}$, which is saturated with respect to $f$, meaning that $f^{-1} f X=X$ (cf. [23, pp. 61, 95] and $[24$, p. 52]).

19 N.B. $e \in K$ will mean that $e$ is a cell of the complex $K$ and $e \subset K, \bar{e} \subset K$, etc., will mean that the sets of points $e, \bar{e}$, etc., are subsets of the space $K$. 
garded as a complex. For example, we may take it to be the complex $K=K^{0}$, which consists entirely of 0 -cells, each point in $K$ being a 0 -cell.

A subcomplex, $L \subset K$, is the union of a subset of the cells of $K$, which are the cells of $L$, such that, if $e \subset L$ then $\bar{e} C L$. Clearly $L$ is a subcomplex if it is the union of a subset of the cells in $K$, which is a closed set of points in $K$. However the above example shows that a subcomplex need not be a closed set of points. Clearly $K^{n}$ is a subcomplex, for each $n \geqq 0$, and we admit the empty set as the subcomplex $K^{-1}$. Also the union and intersection of any set of subcomplexes, finite or infinite, are obviously subcomplexes. If $X \subset K$ is an arbitrary set of points we shall use $K(X)$ to stand for the intersection of all the subcomplexes of $K$, which contain $X$. Obviously $K(p)$ $=K(e)=K(\bar{e})$, where $p$ is any point in $K$ and $e \in K$ is the cell which contains $p$. A finite subcomplex, $L$ (i.e. one which contains but a finite number of cells) is a closed, and indeed a compact subset of $K$. For it is the union of the finite aggregate of compact sets, $\bar{e}$, for each cell $e \in L$.

The topological product, $K_{1} \times K_{2}$, of complexes $K_{1}, K_{2}$ is a complex, whose cells are the products, $e^{n_{1}+n_{2}}=e_{1}^{n_{1}} \times e_{2}^{n_{2}}$, of all pairs of cells $e_{1}^{n_{1}} \in K_{1}, e_{2}^{n_{2}} \in K_{2}$. For let $f_{i}: \sigma^{n_{i}} \rightarrow \bar{e}^{n_{i}}(i=1,2)$ be a characteristic map for $e^{n_{i}}$, let $g: \sigma^{n_{1}} \times \sigma^{n_{2}} \rightarrow \bar{e}^{n_{1}+n_{2}}$ be given by $g\left(p_{1}, p_{2}\right)=\left(f_{1} p_{1}, f_{2} p_{2}\right)$ and let $h: \sigma^{n_{1}+n_{2}} \rightarrow \sigma^{n_{1}} \times \sigma^{n_{2}}$ be a homeomorphism (onto). Then $g h: \sigma^{n_{1}+n_{2}}$ $\rightarrow \bar{e}^{n_{1+n} n_{2}}$ obviously satisfies the conditions (4.1). Therefore $K_{1} \times K_{2}$ is a complex, with this cell structure. In particular $K \times I$ is a complex, which consists of the cells $e \times 0, e \times 1, e \times(0,1)$, for each cell $e \in K$, where $(0,1)$ is the open interval $0<t<1$.

Let $K$ be a locally connected complex, let $\tilde{K}$ be a (locally connected) covering space of $K$ and let $p: \widetilde{K} \rightarrow K$ be the covering map. That is to say there is a basis, $\{U\}$, for the open sets in $K$ such that, if $U \in\{U\}$ then $p$ maps each component of $\boldsymbol{p}^{-1} U$ homeomorphically onto $U$ (cf. $\left[20\right.$, p. 40]). Let $\tilde{x} \in \tilde{K}$ be a given point and let $e^{n} \in K$ be the cell which contains $x=p \tilde{x}$. Then a characteristic map, $f: \sigma^{n} \rightarrow \bar{e}^{n}$, can be "lifted" into a unique map, ${ }^{20} \tilde{f}: \sigma^{n} \rightarrow \tilde{K}$, such that $f=p \tilde{f}$ and $\tilde{f}\left(f^{-1} x\right)=\tilde{x}$. Let $\widetilde{e}^{n}=\tilde{f}\left(\sigma^{n}-\partial \sigma^{n}\right)$ and let $p_{0}=p \mid \tilde{e}^{n}$. Then $f \mid \sigma^{n}-\partial \sigma^{n}=p_{0}\left(\tilde{f} \mid \sigma^{n}-\partial \sigma^{n}\right)$ and since $f \mid \sigma^{n}-\partial \sigma^{n}$ is a (1-1) map onto $e^{n}$ it follows that $p_{0}$ is (1-1) and is onto $e_{n}$. Since $p$, and hence $p_{0}$, is an open mapping it follows that $p_{0}$ is a homeomorphism. Since

$$
\tilde{f} \mid \sigma^{n}-\partial \sigma^{n}=p_{0}^{-1}\left(f \mid \sigma^{n}-\partial \sigma^{n}\right)
$$

${ }^{20}$ See [21, Theorem 2, p. 40] or [22]. We shall sometimes use the same symbol, $f$ or $g$, to denote two maps, $f: A \rightarrow B, g: A \rightarrow C \subset B$, such that $f a=g a$ for each point $a \in A$, even though $B \neq C$. 
it follows that $\tilde{f} \mid \sigma^{n}-\partial \sigma^{n}$ is a homeomorphism, which, according to the definition of $\widetilde{e}^{n}$, is onto $\widetilde{e}^{n}$. Also $\bar{f} \partial \sigma^{n} \subset \tilde{K}^{n-1}=p^{-1} K^{n-1}$. Therefore $\tilde{f}$ satisfies the conditions (4.1). It follows that $\widetilde{K}$ is a complex, each of whose cells is mapped by $p$ homeomorphically onto a cell of $K$.

Let $Q$ be a subcomplex of $\tilde{K}$ and let $e$ be a given cell in $Q$. Then $p \bar{e}$ is closed, since $\bar{e}$ is compact, and $p \bar{e} \subset p Q$. Therefore $\overline{p e}=p \bar{e} \subset p Q$. Therefore $p Q$ is a subcomplex of $K$, which consists of the cells $p e$ for each cell $e \in Q$.

5. CW-complexes. We shall describe a complex, $K$, as closure finite if, and only if, $K(e)$ is a finite subcomplex, for every cell $e \in K$. Since $K(p)=K(e)$ if $p \in e$ this is equivalent to the condition that $K(p)$ is finite for each point $p \in K$. If $L \subset K$ is a subcomplex and $e \in L$ then obviously $L(e)=K(e)$. Therefore any subcomplex of a closure finite complex is closure finite.

We shall say that $K$ has the weak topology (cf. [1, pp. 316, 317]) if, and only if, a subset $X \subset K$ is closed (open) provided $X \cap \bar{e}$ is closed (relatively open) for each cell $e \in K$. If $K$ is closure finite this is equivalent to the condition that $X$ is closed provided $X \cap L$ is closed for every finite subcomplex $L \subset K$. For $X \cap L$ is the union of the finite number of sets $X \cap \bar{e}(e \in L)$. Therefore $X \cap L$ is closed if each set $X \cap \bar{e}$ is closed. Conversely, if $X \cap L$ is closed for each finite subcomplex, $L$, and if $K(\bar{e})$ is finite, then $X \cap \bar{e}$ is closed, since $X \cap \bar{e}$ $=X \cap K(\bar{e}) \cap \bar{e}$.

By a CW-complex we mean one which is closure finite and has the weak topology. Any finite complex, $K$, is obviously closure finite and it has the weak topology since $X \subset K$ is the union of the finite number of sets $X \cap \bar{e}(e \in K)$. Therefore any finite complex is a CWcomplex. Also a complex, $K$, is a CW-complex if it is locally finite, meaning that each point $p \in K$ is an inner point of some finite subcomplex of $K$. For let $K$ be locally finite. Then $K(p)$ is finite, for each point $p \in K$. Therefore $K$ is closure finite. Let $X \subset K$ be such that $X \cap L$ is closed for each finite subcomplex $L \subset K$. Let $L$ be a finite subcomplex of which a given point $p \in K-X$ is an inner point. Since $X \cap L$ is closed, $p$ is an inner point of $L-X=L-(X \cap L)$. Therefore $X$ is closed and $K$ has the weak topology. It may be verified that the number of cells, and hence the number of finite subcomplexes of a connected, locally finite complex, $K$, is countable. Hence, and from (G) below, it may be proved that $K$ is a separable metric space.

If the cells in a CW-complex, $K$, have a maximum dimensionality we call this the dimensionality, $\operatorname{dim} K$, of $K$. If there is no such maximum we write $\operatorname{dim} K=\infty$.

Examples of complexes which are not CW-complexes are: 
(1) $\partial \sigma^{n}(n>1)$ regarded as a "0-dimensional" complex, $K^{0}$, whose cells are the points of $\partial \sigma^{n}$. This is closure finite but does not have the weak topology.

(2) $\sigma^{n}(n>1)$, regarded as a complex $K^{n}=K^{0} \cup e^{n}$, where $e^{n}=\sigma^{n}$ $-\partial \sigma^{n}$ and $K^{0}=\partial \sigma^{n}$, as in (1). This has the weak topology, since $\bar{e}^{n}=K^{n}$, but is not closure finite.

(3) a simplicial complex, which has a metric topology but which is not locally finite (e.g. a complex covering the coordinate axes in Hilbert space). The weak topology in such a complex cannot be metricized (cf. [1, pp. 316, 317]).

Let $K$ be a CW-complex. We establish some properties of $K$.

(A) $A$ map, $f: X \rightarrow Y$, of a closed (open) subset, $X \subset K$, in any space, $Y$, is continuous provided $f \mid X \cap \bar{e}$ is continuous for each cell $e \in K$.

Let $f_{e}=f \mid X \cap \bar{e}$ be continuous, for each cell $e \in K$. Let $Y_{0}$ be any closed (open) subset of $Y$. Obviously $\bar{e} \cap f^{-1} Y_{0}=f_{e}^{-1} Y_{0}$ and it follows from the continuity of $f_{e}$ that $\bar{e} \cap f^{-1} Y_{0}$ is a relatively closed (open) subset of $X \cap \bar{e}$. But $X \cap \bar{e}$ is a closed (relatively open) subset of $\bar{e}$, whence $\bar{e} \cap f^{-1} Y_{0}$ is closed (relatively open) in $\bar{e}$. Therefore $f^{-1} Y_{0}$ is closed (open) in $K$, and a fortiori in $X$. Therefore $f$ is continuous.

(B) $A$ subcomplex, $L \subset K$, is a closed subspace of $K$ and the topology induced by $K$ is the weak topology in $L$.

Let $Y \subset L$ be such that $Y \cap L_{0}$ is closed, and hence compact, for each finite subcomplex $L_{0} \subset L$. Since $Y \cap L_{0}$ is compact it is a closed subset of $K$. Let $K_{0}$ be any finite subcomplex of $K$. Then $L_{0}=L \cap K_{0}$ is a finite subcomplex of $L$ and

$$
Y \cap K_{0}=Y \cap L \cap K_{0}=Y \cap L_{0} .
$$

Therefore $Y \cap K_{0}$ is closed, whence $Y$ is closed in $K$, and a fortiori in $L$. Therefore $L$ has the weak topology. Also, taking $Y=L$, it follows that $L$ is closed, which establishes (B).

(C) If $K$ is connected so is $K^{n}$ for each $n>0$.

Let $n>0$ and let $K^{n}$ be the union of disjoint, nonvacuous closed sets $K_{1}^{n}, K_{2}^{n}$. Since the closure of a cell $e \in K$ is connected it follows that $\bar{e} \subset K_{i}^{n}$ if $e \cap K_{i}^{n} \neq 0(i=1,2)$. Therefore $K_{i}^{n}$ is a subcomplex of $K$. Clearly $\partial e^{n+1}$ is connected $\left(e^{n+1} \in K\right)$, whence it lies either in $K_{1}^{n}$ or in $K_{2}^{n}$. Therefore $K^{n+1}$ is the union of disjoint subcomplexes, $K_{1}^{n+1}$, $K_{2}^{n+1}$, where $K_{i}^{n} \subset K_{i}^{n+1}$ and $e^{n+1} \in K_{i}^{n+1}$ if $\partial e^{n+1} \subset K_{i}^{n}$. A similar (inductive) argument shows that $K^{m}$ is the union of disjoint subcomplexes, $K_{1}^{m}, K_{2}^{m}$, such that $K_{i}^{m} \subset K_{i}^{m+1}(m=n, n+1, \cdots)$. Let $K_{i}$ be the union of the $K_{i}^{m}$ for $m=n, n+1, \cdots$. Then $K_{i} \cap K^{m}=K_{i}^{m}$ and

$$
K_{1} \cap K_{2}=\bigcup_{m}\left(K_{1} \cap K_{2}\right) \cap K^{m}=\bigcup_{m} K_{1}^{m} \cap K_{2}^{m}=0 .
$$


Also $K_{i} \neq 0$, since $K_{i}^{n} \subset K_{i}$, and $K_{i}$ is a closed subset of $K$, according to (B). Therefore $K$ is not connected, which establishes (C).

(D) If $X \subset K$ is compact, then $K(X)$ is a finite complex.

If $X$ meets but a finite number of cells, $e_{1}, \cdots, e_{k} \subset K$, it is contained in the finite union of the (finite) subcomplexes $K\left(e_{1}\right), \cdots$, $K\left(e_{k}\right)$. Assume that there is an infinite set of cells, $\left\{e_{i}\right\}$, each of which meets $X$ and let $p_{i} \in X \cap e_{i}$. Then a finite subcomplex, $L \subset K$, contains but a finite set of the cells in $\left\{e_{i}\right\}$ and $e_{i} \cap L=0$ unless $e_{i} \in L$. Therefore $L$ contains but a finite number of points in the set $P=\left\{p_{i}\right\}$, whence $P$ is closed. Similarly any subset of $P$ is closed, whence $P$ is discrete. But this is absurd, since $P$ is compact, being a closed subset of $X$. Therefore (D) is established.

(E) If a complex $L$, and also $L^{n}$ for each $n \geqq 0$, all have the weak topology, then $L$ is a $C W$-complex.

Certainly $L^{0}$ is closure finite. Assume that $L^{n-1}$ is closure finite, and hence a CW-complex, for some $n>0$. Let $e^{n}$ be a given $n$-cell in $L^{n}$. Since $\partial e^{n}$ is compact it follows from (D) that $L\left(\partial e^{n}\right)$ is finite. But obviously $L\left(e^{n}\right)=L\left(\partial e^{n}\right) \cup e^{n}$ and it follows from induction on $n$ that $L$ is closure finite, which establishes $(\mathrm{E})$.

Let $f: K \rightarrow L$ be a map of $K$ onto a closure finite complex $L$, which has the indentification topology ${ }^{18}$ determined by $f$. Further let the subcomplex $L(f \bar{e})$ be finite for each cell $e \in K$.

(F) Subject to these conditions $L$ is a $C W$-complex.

Let $Y \subset L$ be such that $Y \cap L_{0}$ is closed for each finite subcomplex $L_{0} \subset L$. Let $L_{0} \bar{e}=L(f \bar{e})$ for a given cell $e \in K$. Then $\bar{e} \subset f^{-1} L_{0}$ and

$$
f^{-1} Y \cap \bar{e}=f^{-1} Y \cap \bar{e} \cap \bar{e} \subset\left(f^{-1} Y \cap f^{-1} L_{0}\right) \cap \bar{e} \subset f^{-1}\left(Y \cap L_{0}\right) \cap \bar{e},
$$

since $f^{-1} A \cap f^{-1} B \subset f^{-1}(A \cap B)$ for any sets $A, B \subset L$. But $f^{-1}\left(Y \cap L_{0}\right)$ $\subset f^{-1} Y$. Therefore

$$
f^{-1} Y \cap \bar{e}=f^{-1}\left(Y \cap L_{0}\right) \cap \bar{e} .
$$

Since $Y \cap L_{0}$ is closed it follows that $f^{-1} Y \cap \bar{e}$ is closed. Therefore $f^{-1} Y$ is closed, since $K$ has the weak topology. Since $L$ has the identification topology determined by $f$ it follows that $Y$ is closed. Therefore $L$ has the weak topology. Since $L$ is closure finite by hypothesis this proves $(\mathrm{F})$.

(G) $K$ is a normal space.

Let $X_{1}, X_{2} \subset K$ be disjoint, closed subsets and let $X_{i}^{r}=X_{i} \cap K^{r}$ $(i=1,2 ; r \geqq 0)$. Clearly $K^{0}$ is a discrete set, and hence normal. Let $n>0$ and assume that there are disjoint, relatively open subsets, $U_{1}^{n-1}, \quad U_{2}^{n-1} \subset K^{n-1}$, such that $X_{i}^{n-1} \subset U_{i}^{n-1}$. Then $X_{i} \cap \bar{U}_{j}^{n-1}=0$ $(i, j=1,2 ; i \neq j)$. If $K^{n}=K^{n-1}$ we define $U_{i}^{n}=U_{i}^{n-1}$. Otherwise let 
$f: \sigma^{n} \rightarrow \bar{e}^{n}$ be a characteristic map for a given $n$-cell $e^{n} \in K$ and let

$$
V_{i}=f^{-1} U_{i}^{n-1} \subset \partial \sigma^{n}, \quad Y_{i}=f^{-1} X_{i} \subset \sigma^{n} .
$$

Since $X_{1} \cap X_{2}=0, X_{i} \cap \bar{U}_{j}^{n-1}=0$ we have $Y_{1} \cap Y_{2}=0, Y_{i} \cap \bar{V}_{j}=0$. Let $p_{0}$ be the centroid of $\sigma^{n}$ and let $r, p$ be polar coordinates for $\sigma^{n}(r \in I$, $\left.p \in \partial \sigma^{n}\right)$ such that $(r, p)$ is the point which divides the rectilinear segment $p_{0} p$ in the ratio $r: 1-r$. Let $V_{i}^{\prime} \subset \sigma^{n}$ be the (open) subset, which consists of all points $(r, p)$ with $p \in V_{i}$ and $1-\epsilon<r \leqq 1$, where $0<\epsilon<1$. Since $Y_{i} \cap \bar{V}_{j}=0$ it follows that, if $\epsilon$ is sufficiently small, then $Y_{i} \cap \bar{V}_{j}^{\prime}=0$, which we assume to be the case. Since $f Y_{i} \subset X_{i}, f \partial \sigma^{n}$ $\subset K^{n-1}$ and $X_{i} \cap K^{n-1} \subset U_{i}^{n-1}$ it follows that $Y_{i} \cap \partial \sigma^{n} \subset V_{i}$. Let $V_{i}^{\prime \prime}$ be an $\eta$-neighborhood of $Y_{i}$, defined in terms of a metric for $\sigma^{n}$, where $\eta$ is so small that $V_{1}^{\prime \prime} \cap V_{2}^{\prime \prime}=0, V_{i}^{\prime \prime} \cap \bar{V}_{j}^{\prime}=0$ and $V_{i}^{\prime \prime}$ $\cap\left(\partial \sigma^{n}-V_{i}\right)=0$. Then $V_{i}^{\prime \prime} \cap \partial \sigma^{n} \subset V_{i}$. Let

$$
W_{i}=V_{i}^{\prime} \cup V_{i}^{\prime \prime} \text {. }
$$

Then $Y_{i} \subset W_{i}$ and $W_{1} \cap W_{2}=0$. Obviously $V_{i}^{\prime} \cap \partial \sigma^{n}=V_{i}$, whence

$$
W_{i} \cap \partial \sigma^{n}=V_{i}=f^{-1} U_{i}^{n-1} \text {. }
$$

Since $f: \sigma^{n}-\partial \sigma^{n}$ is a (1-1) map onto $e^{n}$ and $f \partial \sigma^{n} \bigcap e^{n}=0$ it follows that $W_{i}$ is saturated ${ }^{18}$ with respect to $f$. Therefore $f W_{i}$ is a relatively open subset of $\bar{e}^{n}$. From (5.1) we have

$$
f W_{i} \cap K^{n-1}=U_{i}^{n-1} \cap \partial e^{n}
$$

and it follows that $f W_{1} \cap f W_{2}=0$.

Let us write $W_{i}=W_{i}\left(e^{n}\right)$ and let

$$
U_{i}^{n}=U_{i}^{n-1} \cup \underset{e^{n} \in K_{K}}{\bigcup} f W_{i}\left(e^{n}\right) .
$$

Then it follows from (5.2) that $U_{i}^{n} \cap K^{n-1}=U_{i}^{n-1}$ and that

$$
U_{i}^{n} \cap \partial e^{n}=U_{i}^{n-1} \cap \partial e^{n}=f W_{i}\left(e^{n}\right) \cap K^{n-1} .
$$

Also $f W_{i}\left(e^{n}\right) \subset K^{n-1} \cup e^{n}$ and $U_{i}^{n} \cap e^{n}=f W_{i}\left(e^{n}\right) \cap e^{n}$. Therefore

$$
\begin{aligned}
U_{i}^{n} \cap \bar{e}^{n} & =\left(U_{i}^{n} \cap \partial e^{n}\right) \cup\left(U_{i}^{n} \cap e^{n}\right) \\
& =f W_{i}\left(e^{n}\right) \cap\left(K^{n-1} \cup e^{n}\right) \\
& =f W_{i}\left(e^{n}\right) .
\end{aligned}
$$

Therefore $U_{i}^{n}$ is a relatively open subset of $K^{n}$. Obviously $X_{i}^{n} \subset U_{i}^{n}$ and $U_{1}^{n} \cap U_{2}^{n}=0$. Therefore such sets, $U_{i}^{n}$, may be defined inductively for every value of $n$. Let them be so defined and let 


$$
U_{i}=\bigcup_{n} U_{i}^{n}
$$

Since $U_{i}^{n+1} \cap K^{n}=U_{i}^{n}$ it follows by induction on $m>n$ that

$$
U_{i}^{m} \cap K^{n}=U_{i}^{m} \cap K^{m-1} \cap K^{n}=U_{i}^{m-1} \cap K^{n}=U_{i}^{n}
$$

and hence that $U_{i} \cap K^{n}=U_{i}^{n}$. Therefore it follows, first that $U_{i}$ is an open subset of $K$ and second that $U_{1} \cap U_{2}=0$. Obviously $X_{i} \subset U_{i}$, which completes the proof of $(\mathrm{G})$.

(H) If $L$ is a locally finite ${ }^{21}$ complex then $K \times L$ is a $C W$-complex. If $e \in K, e^{\prime} \in L$ are cells in $K$ and $L$ respectively, then the cell $e \times e^{\prime} \in K \times L$ is contained in the finite subcomplex $K(e) \times L\left(e^{\prime}\right)$ $C K \times L$. Therefore $K \times L$ is closure finite.

Let the cells in $K$ be indexed and with each $m$-cell, $e_{i}^{m} \in K$, $(m=0,1, \cdots)$ let us associate an $m$-element, $E_{i}^{m}$, as follows. The points in $E_{i}^{m}$ shall be the pairs $\left(x, e_{i}^{m}\right)$, for every point $x \in \sigma^{m}$, and $E_{i}^{m}$ shall have the topology which makes the map $x \rightarrow\left(x, e_{i}^{m}\right)$ a homeomorphism. No two of these elements have a point in common and we unite them into a topological space,

$$
P=\underset{m, i}{\bigcup} E_{i}^{m}
$$

in which each $E_{i}^{m}$, with its own topology, is both open and closed. Let $f_{i}^{m}: \sigma^{m} \rightarrow \bar{e}_{i}^{m}$ be a characteristic map for $e_{i}^{m}$ and let $\phi: P \rightarrow K$ be the map which is given by $\phi\left(x, e_{i}^{m}\right)=f_{i}^{m} x$, for each point $\left(x, e_{i}^{m}\right) \in P$. Since $\bar{e}_{i}^{m}$ has the identification topology determined by $f_{i}^{m}$ it follows that the weak topology in $K$ is the identification topology determined by $\phi$.

Let a space,

$$
Q=\bigcup_{n, j} E_{j}^{n}
$$

and a map, $\psi: Q \rightarrow L$, be similarly associated with $L$. Then $K \times L$ $=\theta(P \times Q)$, where $\theta: P \times Q \rightarrow K \times L$ is given by $\theta(p, q)=(\phi p, \psi q)$ $(p \in P, q \in Q)$. Also $P \times Q$ is the union of the $(m+n)$-elements $E_{i}^{m} \times E_{j}^{n}$, and $\theta\left(E_{i}^{m} \times E_{j}^{m}\right)=\bar{e}_{i j}^{m+n}$, where $e_{i j}^{m+n}=e_{i}^{m} \times e_{j}^{m}$. Therefore the weak topology in $K \times L$ is obviously the same as the identification topology determined by $\theta$.

Let $V \subset L$ be an open subset and $y \in V$ an arbitrary point in $V$. Since $y$ is an inner point of a finite subcomplex, $L_{0} \subset L$, it is contained in a subset, $V_{0} \subset V \cap L_{0}$, which is open in $L$. Since $L$ is normal there is a neighborhood, $W$, of $y$ such that $\bar{W} \subset V_{0}$. Since $\bar{W} \subset V_{0} \subset L_{0}$ and

\footnotetext{
${ }^{21}$ I do not know if this restriction on $L$ is necessary.
} 
since $V_{0}$ is open in $L$, it follows that $\bar{W} \cap \bar{e}=0$ for any cell $e \in L-L_{0}$. Therefore there are only a finite number of cells in $L$, whose closures meet $\bar{W}$. Therefore $\psi^{-1} \bar{W}$ is contained in the union of a finite subset of the components $E_{i}^{n} \subset Q$. Therefore $\psi^{-1} \bar{W}$ is compact and $(\mathrm{H})$ follows from Lemma 4 in [7].

(I) A homotopy, $f_{t}: X \rightarrow Y$, of a closed (open) subset, $X \subset K$, in an arbitrary space, $Y$, is continuous provided $f_{t} \mid X \cap \bar{e}$ is continuous for each cell $e \in K$.

This follows from (H), with $L=I$, and (A), applied to the subset $X \times I \subset K \times I$ and the map $f: X \times I \rightarrow Y$, which is given by $f(x, t)=f_{t} x$.

(J) (Homotopy extension.) Let $f_{0}: K \rightarrow X$ be a given map of $K$ in an arbitrary space $X$. Let $g_{t}: L \rightarrow X$ be a homotopy of $g_{0}=f_{0} \mid L$, where $L$ is a subcomplex of $K$. Then there is a homotopy, $f_{t}: K \rightarrow X$, such that $f_{t} \mid L=g_{t}$.

Let $K_{r}=L \cup K^{r}\left(r \geqq-1 ; K_{-1}=L\right)$ and assume that $g_{t}$ has been extended to a homotopy, $f_{t}^{n-1}: K_{n-1} \rightarrow X$, such that $f_{0}^{n-1}=f_{0} \mid K_{n-1}$, $f_{t}^{n-1} \mid L=g_{t}(n \geqq 0)$. The homotopy $f_{t}^{n-1}$ can be extended throughout $K_{n-1} \cup e^{n}$, for each $n$-cell $22 e^{n} \in K_{n}-L$, and hence, by (I), to a (continuous) homotopy $f_{t}^{n}: K_{n} \rightarrow X$. Starting with $f_{t}^{-1}=g_{t}$ it follows by induction on $n$ that there is a sequence of homotopies, $f_{t}^{n}: K_{n} \rightarrow X$ $(n=0,1, \cdots)$, such that $f_{0}^{n}=f_{0}\left|K_{n}, f_{t}^{n}\right| K_{n-1}=f_{t}^{n-1}$. It follows from (I) that a homotopy, $f_{t}: K \rightarrow X$, which satisfies the requirements of $(\mathrm{J})$, is given by $f_{t} \mid K_{n}=f_{t}^{n}$.

Let $X_{0} \subset X_{1} \subset \cdots$ be a sequence of subspaces of a given space, $X$, such that any map, $\left(\sigma^{n}, \partial \sigma^{n}\right) \rightarrow\left(X, X_{n-1}\right)$, is homotopic, rel. $\partial \sigma^{n}$, to a $\operatorname{map}^{23} \sigma^{n} \rightarrow X_{n}(n=0,1, \cdots)$. Let $L \subset K$ be a given subcomplex, which may be empty, and let $f_{0}: K \rightarrow X$ be a map such that $f_{0} L^{n} \subset X_{n}$, for each $n=0,1, \cdots$.

(K) There is a homotopy, $f_{t}: K \rightarrow X$, rel. $L$, such that $f_{1} K^{n} \subset X_{n}$ for each $n=0,1, \cdots$.

Since each point in $X$ is joined by an arc to some point in $X_{0}$ there is a homotopy, $f_{i}^{0}: K^{0} \rightarrow X$, rel. $L^{0}$, such that $f_{0}^{0}=f_{0} \mid K^{0}$ and $f_{1}^{0} K^{0} \subset X_{0}$. Let $n>0$ and assume that there is a homotopy $f_{t}^{n-1}: K^{n-1} \rightarrow X$, rel. $L^{n-1}$, such that $f_{0}^{n-1}=f_{0} \mid K^{n-1}, f_{1}^{n-1} K^{n-1} \subset X_{n-1}$. It follows from (J) that $f_{t}^{n-1}$ can be extended, first throughout $L^{n}$ by writing $f_{t}^{n-1} \mid L^{n}$ $=f_{0} \mid L^{n}$, and then to a homotopy, $\xi_{t}: K^{n} \rightarrow X$, rel. $L^{n}\left(\xi_{0}=f_{0} \mid K^{n}\right)$. Since $\xi_{1} K^{n-1} \subset X_{n-1}$ it follows from a standard argument (see [6, §8]), and the condition on $X_{0}, X_{1}, \cdots$, that there is a homotopy, $\eta_{t}: K^{n}$ $\rightarrow X$, rel. $\left(K^{n-1} \cup L^{n}\right)$, such that $\eta_{0}=\xi_{1}, \eta_{1} K^{n} \subset X_{n}$. If $\operatorname{dim} K<\infty$ we

${ }^{22}$ See [5, Lemma 10 in \$16].

${ }^{23}$ If $n=0$ this simply means that each point in $X$ is joined by an arc to some point in $X_{0}$. 
define $f_{t}^{n}: K^{n} \rightarrow X$ as the resultant of $\xi_{t}$ followed by $\eta_{t}$. Then $f_{t}^{n}$ may be defined inductively for every $n \geqq 0$ and we take $f_{t}=f_{t}^{m}$, where $m=\operatorname{dim}$ $K$. But if $\operatorname{dim} K=\infty$ this method fails and we shall define $f_{t}^{n}$ as an extension of $f_{t}^{n-1}$, not as the resultant of $\xi_{t}$ followed by $\eta_{t}$.

If $K^{n}=K^{n-1}$ we define $f_{t}^{n}=f_{t}^{n-1}$. Otherwise let $g: \sigma^{n} \rightarrow \bar{e}^{n}$ be a characteristic map for a given $n$-cell $e^{n} \in K$. Let $r, p$ be polar coordinates for $\sigma^{n}$, defined as in (G), and let $\rho_{t}: \bar{e}^{n} \rightarrow X$ be defined by

$$
\begin{aligned}
\rho_{t} g(r, p) & =\xi_{2 t /(1+r)} g(r, p) & & \text { (if } 0 \leqq 2 t \leqq 1+r) \\
& =\eta_{(2 t-1-r) /(1-r)} g(r, p) & & \text { (if } 1+r<2 t \leqq 2) .
\end{aligned}
$$

Since $\eta_{0}=\xi_{1}$ and $g^{-1} \mid e^{n}$ is a homeomorphism onto $\sigma^{n}-\partial \sigma^{n}$ it follows that $\rho_{t} \mid e^{n}$ is single-valued and continuous. Since $\rho_{t} x=\xi_{t} x$ for any point $x=g(1, p) \in \partial e^{n}$ it follows that $\rho_{t}$ is single-valued. Also $\rho_{t}$ is continuous at $\{g(r, p), t\}$ if $r<1$ and, obviously, if $t<1$. I say that it is continuous at $\{g(1, p), 1\}=(g p, 1)$. For $g p \in K^{n-1}$ and $\eta_{t}\left|K^{n-1}=\eta_{0}\right| K^{n-1}$ $=\xi_{1} \mid K^{n-1}$. Therefore, given a neighborhood, $U \subset X$, of $\xi_{1} g p=\eta_{t} g p$, it follows from the compactness of $I$ that there is a neighborhood, $V \subset \bar{e}^{n}$, of $g p$ such that $\eta_{t} x \in U$ for every $t \in I$, provided $x \in V$. There is also a neighborhood, $V^{\prime} \subset \bar{e}^{n}$, of $g p$, and a $\delta>0$ such that $\xi_{t} x \in U$ if $x \in V^{\prime}, 1-2 \delta<t \leqq 1$. Since $(2-2 \delta) /(1+r)>1-2 \delta$ it follows that $\rho_{t} x \in U$ if $x \in V \cap V^{\prime}, 1-\delta<t \leqq 1$. Therefore $\rho_{t}$ is continuous. Also

$$
\begin{aligned}
& \rho_{t} g(1, p)=\xi_{t} g(1, p)=f_{t}^{n-1} g(1, p), \\
& \rho_{0} g(r, p)=\xi_{0} g(r, p)=f_{0} g(r, p), \\
& \rho_{1} g(r, p)=\eta_{1} g(r, p) \in X_{n} .
\end{aligned}
$$

Therefore a homotopy, $f_{t}^{n}: K^{n} \rightarrow X$, rel. $L^{n}$, such that

$$
f_{t}^{n}\left|K^{n-1}=f_{t}^{n-1}, \quad f_{0}^{n}=f_{0}\right| K^{n}, f_{1} K^{n} \subset X_{n},
$$

is defined by $f_{t}^{n}\left|K^{n-1}=f_{t}^{n-1}, f_{t}^{n}\right| \bar{e}^{n}=\rho_{t}$, for each $n$-cell $e^{n} \in K^{n}$. It follows from induction on $n$ that such a homotopy is defined for each $n \geqq 0$ and a homotopy, $f_{t}: K \rightarrow X$, which satisfies the requirements of $(\mathrm{K})$, is defined by $f_{t} \mid K^{n}=f_{t}^{n}$.

Let $f_{0}: K \rightarrow P$ be a map of $K$ into a CW-complex, $P$, such that $f_{0} \mid L$ is cellular, where $L \subset K$ is a subcomplex. Also let $g_{t}: K \rightarrow P$ be a homotopy such that the maps $g_{0}, g_{1}$ and the homotopy $g_{t} \mid L$ are cellular.

(L) There is a homotopy, $f_{t} \mid K \rightarrow P$, rel. L, of $f_{0}$ into a cellular map $f_{1}$. There is a cellular homotopy, $g_{t}^{\prime}: K \rightarrow P$, such that $g_{0}^{\prime}=g_{0}, g_{1}^{\prime}=g_{1}$, $g_{t}^{\prime \prime}\left|L=g_{t}\right| L$.

Since any continuous image of $\sigma^{n}$ in $P$ is compact it is contained in 
a finite subcomplex $Q \subset P$, according to (D). Any map $\left(\sigma^{n}, \partial \sigma^{n}\right)$ $\rightarrow\left(Q, Q^{n-1}\right)$ is homotopic, ${ }^{24}$ rel. $\partial \sigma^{n}$, in $Q$ to a map $\sigma^{n} \rightarrow Q^{n}$. Therefore the first part follows from $(\mathrm{K})$. The second part follows from the first part with $K, L, f_{0}$ replaced by $K \times I,(K \times 0) \cup(L \times I) \cup(K \times 1)$, $g: K \times I \rightarrow P$, where $g(p, t)=g_{t} p$.

(M) $K$ is locally contractible.

Let $a_{0} \in K$ be a given point, let $U \subset K$ be a given neighborhood of $a_{0}$ and let $e^{r} \in K$ be the cell which contains $a_{0}$. Let $E^{r} \subset U \cap e^{r}$ be an $r$-element, which contains $a_{0}$ in its interior, $V^{r}=E^{r}-\partial E^{r}$, and let $f_{t}^{r}: V^{r} \rightarrow V^{r}$ be a homotopy such that $f_{0}^{r}=1, f_{1}^{r} V^{r}=a_{0}$. Using induction on $n$ we shall define sequences of relatively open subsets, $V^{n} \subset K^{n}$ $(n=r, r+1, \cdots)$, such that $V^{n+1} \cap K^{n}=V^{n}, \bar{V}^{n} \subset U$, and of homotopies $f_{t}^{n}: V^{n} \rightarrow V^{n}$, such that $f_{t}^{n+1} \mid V^{n}=f_{t}^{n}, f_{0}^{n}=1, f_{1}^{n} V^{n}=a_{0}$. Assuming that this has been done, let

$$
V=\bigcup_{n} V^{n}
$$

and let $f_{t}: V \rightarrow V$ be defined by $f_{t} \mid V^{n}=f_{t}^{n}$. Then it follows from the definition of the weak topology and from (I) that $V$ is open in $K$ and $f_{t}$ continuous. Obviously $V \subset U, f_{0}=1, f_{1} V=a_{0}$ and (M) follows.

Assume that $V^{n-1}$ and $f_{t}^{n-1}$ satisfy the above conditions for some $n>r$. Let $g: \sigma^{n} \rightarrow \bar{e}^{n}$ be a characteristic map for a given $n$-cell, $e^{n} \in K$, and let polar coordinates, $r, p$, for $\sigma^{n}$ be defined as in (G). If $g \partial \sigma^{n}$ $\cap V^{n-1}=0$, let $W \subset \sigma^{n}$ be the empty set. Otherwise let $W \subset \sigma^{n}$ be the (open) subset, which consists of all points, $(r, p)$, such that

$$
1-\epsilon<r \leqq 1, \quad p \in g^{-1} V^{n-1},
$$

where $0<\epsilon<1$. Since $\bar{V}^{n-1} \subset U$, whence $g^{-1} \bar{V}^{n-1} \subset g^{-1} U$, it follows that $\bar{W} C g^{-1} U$ if $\epsilon$ is sufficiently small, which we assume to be the case. Let $\xi_{t}: W \rightarrow W$ be the "radial projection," which is defined by

$$
\xi_{t}(r, p)=(r+t-r t, p),
$$

and let $\theta_{t} g W \rightarrow V^{n-1} \cup g W$ be given by

$$
\begin{aligned}
\theta_{t} g(r, p) & =g \xi_{2 t /(1-r)}(r, p) & & \text { (if } 0 \leqq 2 t<1-r) \\
& =f_{(2 t-1+r) /(1+r)}^{n-1} g(1, p) & & \text { (if } 1-r \leqq 2 t \leqq 2) .
\end{aligned}
$$

Since $\xi_{t}(1, p)=(1, p)$ and $g \xi_{1}(r, p)=g(1, p)=f_{0}^{n-1} g(1, p)$ it follows from an argument similar to the one which comes after (5.3) that $\theta_{t}$ is single-valued and continuous. Also

${ }^{24}[5, \S 16$, Theorem 6]. It follows from Theorem 6 in [5] that the condition $f_{t} x \in P\left(f_{0} x\right)(x \in K)$ may be imposed on the homotopy $f_{t}$ in $(L)$. 


$$
\begin{aligned}
& \theta_{t} g(1, p)=f_{t}^{n-1} g(1, p), \\
& \theta_{0} g(r, p)=g \xi_{0}(r, p)=g(r, p), \\
& \theta_{1} g(r, p)=f_{1}^{n-1} g(1, p)=a_{0} .
\end{aligned}
$$

Let $V^{n}$ be the union of $V^{n-1}$ and the sets $g W$, which are thus defined for all the $n$-cells in $K$. Arguments used in (G) show that $V^{n}$ is a relatively open subset of $K^{n}$ and that $V^{n} \cap K^{n-1}=V^{n-1}$. Also it follows from the definition of $W$ that $\partial \sigma^{n} \cap \bar{W} \subset g^{-1} \bar{V}^{n-1}$, whence

$$
K^{n-1} \cap g \bar{W} \subset \bar{V}^{n-1} \text {. }
$$

Hence it follows from the definition of the weak topology that $\bar{V}^{n}$ is the union of $\bar{V}^{n-1}$ and the sets $g \bar{W}$, which are closed since $\bar{W}$ is compact. Since $\bar{V}^{n-1} \subset U, \bar{W} \subset g^{-1} U$ it follows that $\bar{V}^{n} \subset U$. Finally define $f_{t}^{n}: V^{n} \rightarrow V^{n}$ by $f_{t}^{n}\left|V^{n-1}=f_{t}^{n-1}, f_{t}^{n}\right| g W=\theta_{t}$. It follows from (5.4) and from (I) that $f_{t}^{n}$ is single-valued and continuous and that $f_{0}^{n}=1$, $f_{1}^{n} V^{n}=a_{0}$. Therefore (M) follows by induction on $n$.

(N) Any covering complex, $\tilde{K}$, of $K$ is a CW-complex.

Since $\widetilde{K}$ is locally connected, by the definition of a covering space, each of its components is both open and closed and is a covering complex of a component of $K$. A locally connected complex is obviously a $\mathrm{CW}$-complex if, and only if, each of its components is a CW-complex. Therefore (N) will follow when we have proved it in case $K$ and $\tilde{K}$ are connected. We assume that this is so and also, to begin with, that $\tilde{K}$ is a regular covering complex of $K$. That is to say the group, $G$, of covering transformations ${ }^{25}$ in $\tilde{K}$ operates transitively on the set $p^{-1} q$, for any point $q \in K$, where $p: \widetilde{K} \rightarrow K$ is the covering map. We shall describe an open set, $U \subset K$, as an elementary neighborhood if, and only if, each component of $p^{-1} U$ is mapped by $p$ topologically onto $U$. We shall describe an elementary neighborhood in $K$ as a basic neighborhood if, and only if, its closure is contained in an elementary neighborhood. We shall describe a subset of $\tilde{K}$ as a basic neighborhood if and only if it is a component of $p^{-1} U$, where $U$ is a basic neighborhood in $K$. If $\tilde{U} \subset \widetilde{K}$ is a basic neighborhood the component of $p^{-1}(p \tilde{U})$ are the sets $T \tilde{U}$ for every $T \in G$. It follows from the definition of $\widetilde{K}$ and the normality of $K$ that the basic neighborhoods constitute a basis for the open sets, both in $K$ and in $\tilde{K}$.

Let $U \subset K$ be a basic neighborhood and let $V$ be an elementary neighborhood such that $\bar{U} \subset V$. Then the components of $\boldsymbol{p}^{-1} V$ are disjoint open sets in $\tilde{K}$, each of which contains exactly one component of $p^{-1} \bar{U}$. Let $Q \subset p^{-1} \bar{U}$ be a set of points, of which at most

${ }^{25}$ I.e., the group of homeomorphisms, $T: \widetilde{K} \rightarrow \widetilde{K}$, such that $\boldsymbol{p} T=\boldsymbol{p}$. 
one lies in each component of $p^{-1} \bar{U}$. Then $Q$ is a closed discrete set. For if $Q$ has a limit point, $\bar{q}$, then $p \bar{q} \in \bar{U} \subset V$, whence $\bar{q}$ lies in one of the components, $\tilde{V}$, of $p^{-1} V$. But this is absurd, since $\tilde{V}$ contains at most one point of $Q$. Therefore $Q$ is closed and discrete.

Let $\tilde{U} \subset \tilde{K}$ be a basic neighborhood, let $U^{*}$ be its closure ${ }^{26}$ and let $C \subset \tilde{K}$ be compact. I say that only a finite number of the sets $T C$ meet $U^{*}$, where $T \in G$. For if $T C$ meets $U^{*}$ then $C$ meets $T^{-1} U^{*}$. Let $q_{T} \in C \cap T^{-1} U^{*}$. Since $T^{\prime} U^{*} \cap T^{\prime \prime} U^{*}=0$ if $T^{\prime} \neq T^{\prime \prime}$ it follows from the preceding paragraph that the aggregate of points $q_{T}$, for every $T$ such that $U^{*} \cap T C \neq 0$, is a discrete, closed subset of $C$. Since $C$ is compact the set $\left\{q_{T}\right\}$ is finite, which proves our assertion.

We now prove that $\tilde{K}$ has the weak topology. Let $\tilde{X} \subset \tilde{K}$ be a subset such that $\tilde{X} \cap e^{*}$ is closed, for every cell $\tilde{e} \in \tilde{K}$. In order to prove that $\tilde{X}$ is closed it is enough to prove that $\tilde{X} \cap U^{*}$ is closed, where $U^{*}$ is the closure of an arbitrary basic neighborhood $\tilde{U} \subset \tilde{K}$. For this implies that $\tilde{U}-\tilde{X}=\tilde{U}-\left(\tilde{X} \cap U^{*}\right)$ is open, whence it follows that $\tilde{K}-\tilde{X}$ is open. Therefore, to simplify the notation, we assume that $\tilde{X} \subset U^{*}$, where $\tilde{U}$ is a basic neighborhood in $\tilde{K}$. Let $X=p \tilde{X}$ and let $e$ be a given cell in $K$. Then ${ }^{27}$

$$
X \cap \bar{e}=p\left(\tilde{X} \cap p^{-1} \bar{e}\right) .
$$

Let $\tilde{e} \in \tilde{K}$ be a cell which covers $e$. Then $p^{-1} \bar{e}$ consists of the sets $T e^{*}$ for every $T \in G$, and $T e^{*}$ is the closure of the cell $T \tilde{e} \in \tilde{K}$. Since $e^{*}$ is compact it follows from the preceding paragraph that only a finite number of the sets $T e^{*}$, say $T_{1} e^{*}, \cdots, T_{k} e^{*}$, meet $U^{*}$. Let $P_{i}=\tilde{X}$ $\cap T_{i} e^{*}(i=1, \cdots, k)$. Then

$$
X \cap \bar{e}=p\left(\tilde{X} \cap p^{-1} \bar{e}\right)=p\left(P_{1} \cup \cdots \cup P_{k}\right) .
$$

But $P_{i}$ is closed, by the hypothesis concerning $\tilde{X}$, and hence compact, since $T_{i} e^{*}$ is compact. Therefore, $P_{1} \cup \ldots \cup P_{k}$ and hence $X \cap \bar{e}$ are compact. Since the cell $e \in K$ is arbitrary it follows that $X$ is closed. Therefore $p^{-1} X$ is closed. Since $U^{*} \cap T U^{*}=0$ if $T \neq 1$ it follows that

$$
\tilde{X}=U^{*} \cap \bigcup_{T} T \tilde{X}=U^{*} \cap p^{-1} X .
$$

Therefore $\tilde{X}$ is closed and it follows that $\tilde{K}$ has the weak topology.

Since $K^{0}$ is discrete it follows that $\tilde{K}^{0}=p^{-1} K^{0}$ is a discrete set of points. That is to say, $\tilde{K}^{0}$ has the weak topology. If $n>0$ then $K^{n}$ is connected, according to (C), and $\tilde{K}^{n}$ is obviously a covering complex of $K^{n}$. It follows from (L) that the injection homomorphism, $\pi_{1}\left(K^{n}\right)$

${ }^{26}$ We shall denote the closure of a set $\widetilde{P} \subset \widetilde{K}$ by $P^{*}$.

${ }^{27}$ If $f: P \rightarrow Q$ is any map and $A \subset P, B \subset Q$, then $f\left(A \cap f^{-1} B\right)=(f A) \cap B$. 
$\rightarrow \pi_{1}(K)$, is onto, whence $\tilde{K}^{n}$ is connected. Obviously $T \widetilde{K}^{n}=\tilde{K}^{n}$ for any $T \in G$ and it follows that $\tilde{K}^{n}$ is a regular covering complex of $K^{n}$. Therefore $\tilde{K}^{n}$ has the weak topology, according to what we have just proved. It follows from (E) that $\widetilde{K}$ is a CW-complex.

Now let $\tilde{K}$ be a (connected) covering complex of $K$, which is not regular. Then a universal covering complex, $\widehat{K}$ of $\widetilde{K}$ is a universal covering complex of $K$. Therefore $\widehat{K}$ is a CW-complex. Let $p: \widehat{K} \rightarrow \widetilde{K}$ be the covering map. Since $p$ is an open map it follows that $\widetilde{K}$ has the identification topology determined by $\boldsymbol{p}$. It follows from the final paragraph in $\S 4$ that $\tilde{K}$ is closure finite and that the remaining condition of $(F)$ is satisfied. Therefore it follows from $(F)$ that $\tilde{K}$ is a $\mathrm{CW}$-complex, which completes the proof of $(\mathrm{N})$.

6. Proof of Theorems 1-4. Let $X, Y$ be spaces in the class $\alpha$ and let $f: X \rightarrow Y$ be a map such that the induced homomorphism, $f_{n}: \pi_{n}(X)$ $\rightarrow \pi_{n}(Y)$, is an isomorphism onto if $1 \leqq n<N+1$, where $N$ $=\max (\Delta X, \Delta Y)$. Let $P$ and $Q$ be $C W$-complexes, of dimensionalities $\Delta X$ and $\Delta Y$, which dominate $X$ and $Y$ respectively. Let $\lambda: X \rightarrow P$, $\lambda^{\prime}: P \rightarrow X$ and $\mu: Y \rightarrow Q, \mu^{\prime}: Q \rightarrow Y$ be maps such that $\lambda^{\prime} \lambda \simeq 1, \mu^{\prime} \mu \simeq 1$. Let $Z$ be the mapping cylinder ${ }^{28}$ of $f$ and $R$ the mapping cyclinder of the map $\mu f \lambda^{\prime}: P \rightarrow Q$, assuming that no two of the spaces $X, Y$, $X \times I$, etc., have a point in common.

Assume that there is a homotopy, $h_{t}:(Z, X) \rightarrow(Z, X)$, such that $h_{0}=1, h_{1} Z \subset X$. Let $h: Z \rightarrow X$ be given by $h z=h_{1} z(z \in Z)$ and let $i: X \rightarrow Z$ be the identical map. Then $i h=h_{1}: Z \rightarrow Z$, whence $i h \simeq 1$. Also $h i \simeq 1$, since $k_{t}: X \rightarrow X$, given by $k_{t} x=h_{t} x(x \in X)$, is a deformation of $k_{0}=1$ into $k_{1}=h i$. Therefore $i$ is a homotopy equivalence. As in [6] it follows that $f: X \rightarrow Y$ is a homotopy equivalence.

We now prove the existence of the homotopy $h_{t}:(Z, X) \rightarrow(Z, X)$. The argument in [6], which refers to the homotopy sequence

$$
\pi_{n}(X) \rightarrow \pi_{n}(Z) \rightarrow \pi_{n}(Z, X) \rightarrow \pi_{n-1}(X) \rightarrow \pi_{n-1}(Z),
$$

shows that $\pi_{n}(Z, X)=0$ if $1 \leqq n<N+1$. Since $\operatorname{dim} Q \leqq N$ it follows from $(\mathrm{K})$, in $\$ 5$ above, that there is a homotopy, $\rho_{t}: Q \rightarrow Z$, such that $\rho_{0}=j \mu^{\prime}, \rho_{1} Q \subset X$, where $j$ is the identical map $j: Y \rightarrow Z$. Therefore $j \simeq j \mu^{\prime} \mu \simeq \rho_{1} \mu$. Since $\rho_{1} \mu Y \subset \rho_{1} Q \subset X$, the argument used in [6] shows that $\pi_{N+1}(Z, X)=0$, in case $N<\infty$.

Let $K=(P \times I) \cup Q$ and let $R$ be formed from the CW-complex $K$ by identifying ${ }^{28}(p, 0)$ with $p$ and $(p, 1)$ with $\mu f \lambda^{\prime} p \in Q$ for each point $p \in P$. Let $\phi: K \rightarrow R$ be given by $\phi(p, 0)=p, \phi(p, 1)=\mu f \lambda^{\prime} p$, $\phi(p, t)=(p, t)$ if $0<t<1, \phi \mid Q=1$. The arguments in [6, §2] show that

${ }^{28}$ See $\$ 7$ below. 
there are maps

$$
\nu:(Z, X) \rightarrow(R, P), \quad \nu^{\prime}:(R, P) \rightarrow(Z, X),
$$

with $\nu^{\prime} \mid Q=j \mu^{\prime}$, and a homotopy, $\zeta_{t}:(Z, X) \rightarrow(Z, X)$, such that $\zeta_{0}=\nu^{\prime} \nu, \zeta_{1}=1$. Let $L=(P \times 0) \cup(P \times 1) \cup Q \subset K$, let $\rho_{t}: Q \rightarrow Z$ mean the same as before and let $\rho_{t}^{\prime}: L \rightarrow Z$ be given by

$$
\begin{aligned}
\rho_{t}^{\prime}(p, 0) & =\nu^{\prime} \phi(p, 0), \\
\rho_{t}^{\prime}(p, 1) & =\rho_{t} \phi(p, 1), \\
\rho_{t}^{\prime} q & =\rho_{t} \phi q \quad(p \in P, q \in Q) .
\end{aligned}
$$

Then $\rho_{0}^{\prime}=\nu^{\prime} \phi \mid L$, since $\rho_{0}=j \mu^{\prime}=\nu^{\prime} \mid Q$, and $\rho_{1}^{\prime} L \subset X$. Let $\psi_{0}=\nu^{\prime} \phi: K \rightarrow Z$. Then it follows from $(\mathrm{J})$ that $\rho_{t}^{\prime}$ has an extension $\psi_{t}: K \rightarrow Z$. Since $\psi_{1} L=\rho_{1}^{\prime} L \subset X$ and since $\operatorname{dim} K \leqq N+1$ and $\pi_{n}(Z, X)=0$ if $1 \leqq n$ $<N+2$ it follows from $(\mathrm{K})$ that there is a homotopy, $\psi_{t}^{\prime}: K \rightarrow Z$, rel. $L$, such that $\psi_{0}^{\prime}=\psi_{1}, \psi_{1}^{\prime} K \subset X$. Let $\theta_{t}: K \rightarrow Z$ be the resultant of $\psi_{t}$ followed by $\psi_{t}^{\prime}$. Then it is easily verified that $\chi_{t}=\theta_{t} \phi^{-1}: R \rightarrow Z$ is single-valued, and hence continuous $[7, \S 5]$. Moreover $\chi_{0}=\psi_{0} \phi^{-1}$ $=\nu^{\prime} \phi \phi^{-1}=\nu^{\prime}$ and $\chi_{1} R=\psi_{1}^{\prime} \phi^{-1} R \subset X$. Therefore the required homotopy, $h_{t}:(Z, X) \rightarrow(Z, X)$, may be defined as the resultant of $\zeta_{1-t}$ followed by $\chi_{t} \nu$. This proves Theorem 1 .

The proofs of Theorems 2, 3, 4 are the same as those of the corresponding theorems in [6].

7. Note on $n$-homotopy. Let us return to Fox's original definition of $n$-homotopy, in terms of finite polyhedra. It is not certain, for reasons indicated in [6], that an $n$-homotopy equivalence, $f: X \rightarrow Y$, induces an isomorphism of $\pi_{r}(X)$ onto $\pi_{r}(Y)(r \leqq n)$, where $X, Y$ are arbitrary, arcwise connected spaces. This question reduces to the following. Let $h_{r}: \pi_{r}(X) \rightarrow \pi_{r}(X)$ be the endomorphism induced by a map, $h: X \rightarrow X$, such that $h \simeq_{n} 1$. Is $h_{r}$ an automorphism if $1 \leqq r \leqq n$ ? Consider the case $r=1$. It is easily proved that, given any finite set of elements, $a_{1}, \cdots, a_{k} \subset \pi_{1}(X)$, there is an element, $\eta \in \pi_{1}(X)$, such that $h_{1} a_{i}=\eta a_{i} \eta^{-1}(i=1, \cdots, k)$. It follows that $h_{1}$ is an isomorphism into. Also it is an inner automorphism if $\pi_{1}(X)$ has a finite set of generators. Is $h_{1}$ an automorphism if $\pi_{1}(X)$ is arbitrary?

Meanwhile we show that these questions can be answered with our definition. Let $X$ be an arbitrary arcwise connected space and let a point $x_{0} \in X$ be chosen as base point for all the groups $\pi_{r}(X)$. We construct a CW-complex, $K=K\left(X, x_{0}\right)$, which is somewhat analogous to the singular complex of homology theory. Let $K^{0}$ consist of a single 0 -cell $e^{0}$. With each map, $\theta:\left(\sigma^{r}, \partial \sigma^{r}\right) \rightarrow\left(X, x_{0}\right)(r=1,2, \cdots)$, we associate a cell, $e_{\theta}^{r} \in K$, such that $\partial e_{\theta}^{r}=e^{0}$. The points of $e_{\theta}^{r}$ are the 
pairs $(p, \theta)$, for each point $p \in \sigma^{r}-\partial \sigma^{r}$, and $g: \sigma^{r} \rightarrow \bar{e}_{\theta}^{r}$, given by $g p=(p, \theta)\left(p \in \sigma^{r}-\partial \sigma^{r}\right), g \partial \sigma^{r}=e^{0}$, is a characteristic map for $e_{\theta}^{r}$. The complex thus defined is obviously closure finite and we give it the weak topology.

Let $f, f^{\prime}: X \rightarrow Y$ be maps of $X$ in any arcwise connected space $Y$, and let $f_{r}, f_{r}^{\prime}: \pi_{r}(X) \rightarrow \pi_{r}(Y)$ be the homomorphisms induced by $f, f^{\prime}$, when the base-point $y_{0} \in Y$ is joined to $f x_{0}, f^{\prime} x_{0}$ by paths (see [12] and $\left[1\right.$, p. 279]) $\lambda, \lambda^{\prime}:(I, 0) \rightarrow\left(Y, y_{0}\right)$. If $\eta \in \pi_{1}(Y), b \in \pi_{r}\left(Y, y_{0}\right)(r \geqq 1)$ we shall use $\eta \cdot b$ to denote the image of $b$ in the automorphism of $\pi_{r}\left(Y, y_{0}\right)$, which is determined by $\eta$. Thus $\eta \cdot b=\eta b \eta^{-1}$ if $r=1$. Using our definition of $n$-homotopy we have:

TheOREM 11. If $f \simeq_{n} f^{\prime}$ there is a fixed element, $\eta \in \pi_{1}(Y)$, such that $f_{r}^{\prime} a=\eta \cdot f_{r} a$ for every $a \in \pi_{r}(X)$ and every $r=1, \cdots, n$.

Let $K=K\left(X, x_{0}\right)$ mean the same as before and let $\phi: K \rightarrow X$ be given by $\phi e^{0}=x_{0}, \phi(p, \theta)=\theta p$, for each point $(p, \theta) \in K-e^{0}$. It follows from (A) in $\S 5$ that $\phi$ is continuous. The homomorphism, $\phi_{r}: \pi_{r}(K) \rightarrow \pi_{r}(X)$, induced by $\phi$ is obviously onto. Since $f \simeq_{n} f^{\prime}$ there is a homotopy, $\xi_{t}: K^{n} \rightarrow Y$, such that $\xi_{0}=f \phi\left|K^{n}, \xi_{1}=f^{\prime} \phi\right| K^{n}$. Let $\mu: I \rightarrow Y$ be given by $\mu(t)=\xi_{t} e^{0}$. Then $\mu(0)=\lambda(1), \mu(1)=\lambda^{\prime}(1)$. Let $\eta \in \pi_{1}(Y)$ be the element represented by the circuit, which consists of $\lambda^{\prime}$, followed by $\mu^{-1}$, given by $\mu^{-1}(t)=\mu(1-t)$, followed by $\lambda^{-1}$. Then it is easily verified that $f_{r}^{\prime} \phi_{r} a=\eta \cdot f_{r} \phi_{r} a$, where $a \in \pi_{r}(K)$ and $1 \leqq r \leqq n$. Since $\phi_{r}$ is onto this proves the theorem.

8. A process of identification. We shall prove a theorem, which is analogous to Theorem 2 in [7]. For this we shall need a lemma concerning a process of identification, which is often used (e.g., in constructing mapping cylinders, attaching cells $k$ to complexes, etc.) but which, as far as I know, has never been described in full generality and detail.

Let $X$ and $Y_{0}$ be given topological spaces and let $f_{0}: X_{0} \rightarrow Y_{0}$ be a map of a closed subset, $X_{0} \subset X$, into $Y_{0}$. We allow $X$ and $Y_{0}$ to have common points, but only if $X \cap Y_{0} \subset X_{0}, X \cap Y_{0}$ being a closed subset, both of $X_{0}$ and of $Y_{0}$, and $f_{0} \mid X_{0} \cap Y_{0}=1$. Let $Y$ be the space which consists of the points in $X-X_{0}$ and in $Y_{0}$, with the identification topology determined by the $\operatorname{map}^{29} \phi: X \cup Y_{0} \rightarrow Y$, where $\phi \mid X-X_{0}=1$, $\phi\left|Y_{0}=1, \phi\right| X_{0}=f_{0}$. Notice that a set $B_{0} \subset Y_{0}$ is closed in $Y$ if, and

${ }^{29} X \cup Y_{0}$ denotes the space, which consists of the points in $X$ and in $Y_{0}$ and in which a subset, $A$, is closed if, and only if, $A \cap X$ and $A \cap Y_{0}$ are closed subsets of the spaces $X$ and $Y_{0}$. Since $X \cap Y_{0}$ is closed in both $X$ and $Y_{0}$ it follows that $X$ and $Y_{0}$ keep their own topologies in $X \cup Y_{0}$. 
only if, $Y_{0} \cap \phi^{-1} B_{0}\left(=B_{0}\right)$ and $X \cap \phi^{-1} B_{0}\left(=f_{0}^{-1} B_{0}\right)$ are both closed in $X \cup Y_{0}$. Since $X_{0}$ is closed it follows from the continuity of $f_{0}$ that $f_{0}^{-1} B_{0}$ is closed in $X$ if $B_{0}$ is closed in $Y_{0}$. Therefore $Y_{0}$ keeps its own topology and is a closed subset of $Y$. Similarly $X-X_{0}$ keeps its own topology. We shall say that $Y$ is formed by attaching $X$, or $X-X_{0}$ to $Y_{0}$ by means of the map $f_{0}$. We shall also say that $Y$ is formed by identifying each point $x \in X_{0}$ with $f_{0} x \in Y_{0}$.

In some cases we may be given the spaces $X, Y$ and a map, $f:\left(X, X_{0}\right) \rightarrow\left(Y, Y_{0}\right)$, where $X_{0} \subset X$ and $Y_{0} \subset Y$ are closed subsets, subject to the conditions:

(8.1) (a) $f \mid X-X_{0}$ is a homeomorphism onto $Y-Y_{0}$;

(b) a subset $B \subset Y$ is closed (open) if, and only if, $B \cap Y_{0}$ is closed (relatively open) and if $f^{-1} B$ is closed (open).

In this case we may always regard $Y$ as formed by attaching a homeomorph of $X$ to $Y_{0}$. For let $h_{0}: X_{0} \rightarrow X_{0}^{\prime}$ be a homeomorphism of $X_{0}$ onto a new space, $X_{0}^{\prime}$, which does not meet $Y$. Let $X^{\prime}$ be the space consisting of the points in $X_{0}^{\prime}$ and in $Y-Y_{0}$ with the topology which makes $h:\left(X, X_{0}\right) \rightarrow\left(X^{\prime}, X_{0}^{\prime}\right)$ a homeomorphism, where $h \mid X_{0}$ $=h_{0}, h\left|X-X_{0}=f\right| X-X_{0}$. The conditions (8.1) are satisfied by $X^{\prime}$, $Y$ and the map $f h^{-1}:\left(X^{\prime}, X_{0}^{\prime}\right) \rightarrow\left(Y, Y_{0}\right)$. Since $X^{\prime}-X_{0}^{\prime}=Y-Y_{0}$ and $f h^{-1} \mid X^{\prime}-X_{0}^{\prime}=1$ it follows that $Y$ is the result of attaching $X^{\prime}$ to $Y_{0}$ by means of the map $f h^{-1} \mid X_{0}^{\prime}$.

A particularly important application of this construction is that of the mapping cylinder of a map, $g: A \rightarrow B$, where $A, B$ are disjoint spaces. After replacing $A \times I$ by a homeomorph, if necessary, we assume that it has no point in common with $A$ or $B$. Then the mapping cylinder is defined as above, with $Y_{0}=A \cup B, X=A \times I$, $X_{0}=(A \times 0) \cup(A \times 1)$ and $f_{0}(a, 0)=a, f_{0}(a, 1)=g a$.

Let $X, Y$ and $f:\left(X, X_{0}\right) \rightarrow\left(Y, Y_{0}\right)$ satisfy (8.1) and let $X-X_{0}$ $=Y-Y_{0}, f \mid X-X_{0}=1$. Let $X$ be a normal, Hausdorff space and let $Y_{0}$ satisfy the following "weak regularity" condition: distinct points $y_{1}, y_{2} \subset Y_{0}$ have neighborhoods $V_{1}, V_{2} \subset Y_{0}$ such that $\bar{V}_{1} \cap \bar{V}_{2}=0$.

Lemma 1. Under these conditions $Y$ is a Hausdorff space.

Let $y_{1}, y_{2}$ be distinct points in $Y$. First assume that at least one of them, say $y_{1}$, is in $Y-Y_{0}=X-X_{0}$. Since $Y_{0}$ and $X$ are Hausdorff spaces, the sets $y_{1}, y_{2}$ and $f^{-1} y_{2}$ are closed. Since $X$ is normal there is therefore a neighborhood, $U \subset X$, of $y_{1}$, such that

$$
\bar{U} \subset X-\left(X_{0} \cup f^{-1} y_{2}\right) .
$$

Then $f \bar{U}=\bar{U} \subset Y-Y_{0}$ and $y_{1} \in U, y_{2} \in Y-\bar{U}$. Also $U$ is open and $\bar{U}$ closed in $Y$. Therefore $U, Y-\bar{U}$ are disjoint, open subsets of $Y$, 
which contain $y_{1}, y_{2}$ respectively.

Let $y_{1}, y_{2} \subset Y_{0}$ and let $V_{1}^{0}, V_{2}^{0} \subset Y_{0}$ be neighborhoods of $y_{1}, y_{2}$ such that $\bar{V}_{1}^{0} \cap \bar{V}_{2}^{0}=0$. Let $U_{i}^{0}=f^{-1} V_{i}^{0} \subset X_{0}(i=1,2)$. Then $\bar{U}_{i}^{0} \subset f^{-1} \bar{V}_{\imath}^{0}$, whence $\bar{U}_{1}^{0} \cap \bar{U}_{2}^{0}=0$. Since $X$ is normal there are disjoint open sets, $U_{1}^{\prime}, U_{2}^{\prime} \subset X$, such that $\bar{U}_{i}^{0} \subset U_{i}^{\prime}$. Let

$$
U_{i}=U_{i}^{\prime}-\left(X_{0}-U_{i}^{0}\right) .
$$

Then $U_{i}$ is open, since $X_{0}-U_{i}^{0}$ is closed, and $U_{1} \cap U_{2}=0, U_{i} \cap X_{0}=U_{i}^{0}$. Let

$$
V_{i}=\left(U_{i}-U_{i}^{0}\right) \cup V_{i}^{0} \subset Y .
$$

Since $U_{i}-U_{i}^{0} \subset Y-Y_{0}$ it follows that $Y_{0} \cap V_{i}=V_{i}^{0}$, which is open in $Y_{0}$. Also

$$
f^{-1} V_{i}=f^{-1}\left(U_{i}-U_{i}^{0}\right) \cup f^{-1} V_{i}^{0}=\left(U_{i}-U_{i}^{0}\right) \cup U_{i}^{0}=U_{i} .
$$

Therefore $V_{i}$ is open in $Y$. Also $y_{i} \in V_{i}$ and, obviously, $V_{1} \cap V_{2}=0$. This completes the proof.

Now let $X$ and $Y_{0}$ be disjoint CW-complexes and let $X_{0}$ be a subcomplex of $X$. Since $X, Y_{0}$ are normal, Hausdorff spaces it follows from Lemma 1 that $Y$ is a Hausdorff space. Let $g: \sigma^{n} \rightarrow \bar{e}^{n}$ be a characteristic map for a given cell $e^{n} \in X-X_{0}$. Then $f g \mid \sigma^{n}-\partial \sigma^{n}$ is a homeomorphism onto $f e^{n}$, where $f: X \rightarrow Y$ means the same as before. It follows that $Y$ is a complex, consisting of the cells in $Y_{0}$ and the cells $f e\left(e \in X-X_{0}\right)$, provided ${ }^{30} f\left(X_{0} \cap \partial e^{n}\right) \subset Y_{0}^{n-1}$ for each $n$-cell $e^{n} \in X-X_{0}$ $(n=0,1, \cdots)$. This being so, I say that $Y$ is closure finite. For any cell $e \in Y_{0}$ is contained in the finite subcomplex $Y_{0}(e)$. Let $e$ be a given cell in $X-X_{0}$, let $K=X(e)$ and let $K_{0}=K \cap X_{0}$. Then $K_{0}$, and hence $f K_{0}$, is compact, and it follows from (D) in $\$ 5$ that $L_{0}=Y_{0}\left(f K_{0}\right)$ is finite. The subset $L_{0} \cup_{f K}$ is compact and is the union of the cells in $L_{0}$ and in $f\left(K-K_{0}\right)$. It is therefore a subcomplex. Moreover it is finite and contains $f e$. Therefore $Y$ is closure finite. Also $Y$ has the identification topology, which is determined by the map $\phi: X \cup Y_{0} \rightarrow Y$, where $\phi|X=f, \phi| Y_{0}=1$. Obviously $X \cup Y_{0}$ is a CW-complex, consisting of the cells in $X$ and in $Y_{0}$, and the cells in $Y$ are the cells $\phi e$ for each cell $e \in\left(X-X_{0}\right) \cup Y_{0}$. Since $Y$ is closure finite it follows from this and from (D) in $\S 5$ that $Y(\phi \bar{e})$ is finite for any $e \in X \cup Y_{0}$. Therefore $Y$ is a $C W$-complex, according to $(\mathrm{F})$ in $\$ 5$. Under these conditions $^{31}$ we say that $Y-Y_{0}$ is isomorphic to $X-X_{0}$ and that $f \mid X-X_{0}$ is an isomorphism of $X-X_{0}$ onto $Y-Y_{0}$.

${ }^{30}$ E.g., if $f_{0}: X_{0} \rightarrow Y_{0}$ is cellular or if $X^{n-1} \subset X_{0}, f X_{0} \subset Y_{0}^{n-1}$.

${ }^{31}$ In this definition we allow $X_{0}$ and $Y_{0}$ to be empty. 
We mention two applications of the preceding paragraph. The first is that the mapping cylinder of a cellular map, $f: K \rightarrow L$, where $K, L$ are $\mathrm{CW}$-complexes, is itself a CW-complex. Secondly, let $X$ be the union of a set of $n$-elements, $\left\{E_{i}^{n}\right\}$, which are disjoint from each other and from $Y_{0}$, each $E_{i}^{n}$ being a subcomplex, $E_{i}^{n}=e_{i}^{0} \cup e_{i}^{n-1} \cup e_{i}^{n}$. Let $X_{0}$ be the union of the boundaries, $\partial E_{i}^{n}=e_{i}^{0} \cup e_{i}^{n-1}$, and let $f_{0}$ be any map of the form $f_{0}: X_{0} \rightarrow Y_{0}^{n-1}$. Then we say that $Y$ is formed by attaching the set of cells $\left\{e_{i}^{n}\right\}$ to $Y_{0}$ by means of the maps $f_{0} \mid \partial E_{i}^{n}$.

Let $X, Y$ and $f:\left(X, X_{0}\right) \rightarrow\left(Y, Y_{0}\right)$ satisfy (8.1), where $X, Y$ are CW-complexes, $X_{0}, Y_{0}$ are subcomplexes and the map $f$ is cellular. Let $f_{0}: X_{0} \rightarrow Y_{0}$ be the map given by $f_{0} x=f x\left(x \in X_{0}\right)$. Then we have:

Theorem 12. If $f_{0}: X_{0} \rightarrow Y_{0}$ is a homotopy equivalence, so is $f: X \rightarrow Y$.

The proof is the same ${ }^{32}$ as that of Theorem 2 in [7].

If $Y_{0}$ is a single point we describe the above process of identification as shrinking $X_{0}$ into the point $Y_{0}$. It follows from Theorems 1 and 12 that a simply connected, aspherical subcomplex, $X_{0} \subset X$, may be shrunk into a point without altering the homotopy type of $X$.

Let $X_{0}=X^{n}$ and let $Y_{0}=Y_{0}^{n}$. Then $Y^{n}=Y_{0}^{n}$ and we have:

Corollary 1. Given a CW-complex, $Y_{0}^{n} \equiv X^{n}$, there is a CW-complex, $Y$, such that $Y^{n}=Y_{0}^{n}, X \equiv Y$ and $Y-Y^{n}$ is isomorphic to $X-X^{n}$. Then

Let $X$ and $Y_{0}$ be connected, let $Y_{0}=Y_{0}^{n}$ and let $Y_{0}^{n} \equiv_{n-1} X^{n}(n \geqq 2)$.

$$
X^{n} \cup \Sigma_{1}^{n} \equiv Y_{0}^{n} \cup \Sigma_{2}^{n}
$$

where $\Sigma_{1}^{n}, \Sigma_{2}^{n}$ mean the same as in Theorem 6. Let us "fill up" the closure, $S^{n}$, of each cell $e^{n} \in \Sigma_{1}^{n}$ with an $(n+1)$-cell, $e^{n+1}$, in such a way that $\bar{e}^{n+1}$ is an $(n+1)$-element bounded by $S^{n}$. The result is a CWcomplex, ${ }^{33} K=X \cup \Sigma_{1}^{n} \cup\left\{e^{n+1}\right\}$. There is obviously a retracting deformation of $K$ into $X$ in which each $(n+1)$-element, $\bar{e}^{n+1}$, is retracted into the point $X \cap \bar{e}^{n+1}$. Therefore $K \equiv X$. Also $K^{n}=X^{n} \cup \Sigma_{1}^{n}, K^{n-1}$ $=X^{n-1}$. Let $L^{n}=Y_{0}^{n} \cup \Sigma_{2}^{n}$. Then $L^{n-1}=Y_{0}^{n-1}$. Therefore, replacing $X, X^{n}, Y_{0}^{n}$ in Corollary 1 by $K, K^{n}, L^{n}$, we have:

Corollary 2. If $Y_{0}^{n} \equiv_{n-1} X^{n}$ there is a CW-complex, $Y$, such that $X \equiv Y$ and $Y^{n-1}=Y_{0}^{n-1}$.

For example, if $\pi_{r}(X)=0$ for $r=1, \cdots, n-1$, we may take $Y$

${ }^{32}$ Notice that $\mathrm{CW}$-complexes and subcomplexes have all the homotopy extension properties required by [10].

${ }^{33}$ We assume that the cells in $\Sigma_{1}^{n}$ and the new $(n+1)$-cells, $\left\{e^{n+1}\right\}$, are disjoint from each other and from $X$. 
to be a single point, as observed in $\S 3$. Then $X \equiv Y$, where $Y^{n-1}=Y_{0}^{n}$ and $Y^{n}$ consists of the $n$-spheres, which are the closures of the $n$-cells in $\Sigma_{2}^{n}$.

9. Countable complexes. We describe a complex as countable if, and only if, the number of its cells is countable. By a locally finite (finite) polyhedron we mean a space which is covered by a locally finite (finite) simplicial complex. The purpose of this section is to prove:

Theorem 13. Any countable (finite), p-dimensional CW-complex $(p \leqq \infty)$ is of the same homotopy type as a locally finite (finite), $p$ dimensional polyhedron.

We shall need three lemmas for the proof of this. A CW-complex, $K^{\prime}$, will be described as a subdivision of a CW-complex $K$ if, and only if, both complexes cover the same space and if each cell of $K^{\prime}$ is contained in a cell of $K$. If $K^{\prime}$ is a simplicial complex we shall describe it as a simplicial subdivision of $K$. Let $K^{\prime}$ be a simplicial subdivision of $K$ and let a set of disjoint $n$-cells, $\left\{e_{i}^{n}\right\}$, be attached to $K$ by maps, $g_{i}: \partial E_{i}^{n} \rightarrow K^{n-1}\left(K \cap E_{i}^{n}=0\right)$, each of which is simplicial with respect to $K^{\prime}$ and some triangulation of $\partial E_{i}^{n}$. Then we have:

Lemma 2. The complex $K \cup\left\{e_{i}^{n}\right\}$ has a simplicial subdivision, of which $K^{\prime}$ is a subcomplex.

This will be proved in $\$ 10$ below.

Let $K$ and $\left\{e_{i}^{n}\right\}$ mean the same as in Lemma 2, except that $K$ need not have a simplicial subdivision. Let $f: K \rightarrow L$ be a homotopy equivalence of $K$ into a CW-complex $L$. Let

$$
h_{i} \simeq f g_{i}: \partial E_{i}^{n} \rightarrow L,
$$

where $h_{i} \partial E_{i}^{n} \subset L^{n-1}$ but $\simeq$ indicates homotopy in $L$. Let the cells $\left\{e_{i}^{n}\right\}$ be attached to $L$ by the maps $h_{i}$, thus forming a complex $L \cup\left\{e_{i}^{n}\right\}\left(L \cap E_{i}^{n}=0\right)$.

LEMMA 3. The map $f: K \rightarrow L$ can be extended to a homotopy equivalence $h: K \cup\left\{e_{i}^{n}\right\} \rightarrow L \cup\left\{e_{i}^{n}\right\}$.

If $h_{i}=f g_{i}$ this follows from Theorem 12, taking $h \mid e_{i}^{n}=1$. In general it follows from Theorem 12 and the proof of Lemma 5 in [5].

Let a connected, CW-complex, $K$, be the union of an "expanding" set of subcomplexes, $K_{0} \subset K_{1} \subset \cdots$, such that if $n>0$ then $K_{n}$ is connected and the injection homomorphism

$$
i_{n}: \pi_{n}\left(K_{n+1}\right) \rightarrow \pi_{n}(K)
$$


is an isomorphism onto (e.g., $\left.K_{n}=K^{n}\right)$. Let $L$ and $L_{0} \subset L_{1} \subset \cdots \subset L$ satisfy the same conditions and let $\left\{K_{n}\right\},\left\{L_{n}\right\}$ be related by a sequence of homotopy equivalences,

$$
f^{n}:\left(K_{n}, K_{n-1}\right) \rightarrow\left(L_{n}, L_{n-1}\right),
$$

such that $f^{n} \mid K_{n-1}=f^{n-1}$.

LEMma 4. The map, $f: K \rightarrow L$, which is given by $f \mid K_{n}=f^{n}$, is a homotopy equivalence.

Let $i_{n}$ mean the same in $L$ as in $K$ and let

$$
f_{n}: \pi_{n}(K) \rightarrow \pi_{n}(L), \quad f_{n}^{n+1}: \pi_{n}\left(K_{n+1}\right) \rightarrow \pi_{n}\left(L_{n+1}\right)
$$

be the homomorphisms induced by $f, f^{n+1}$. Then $f_{n}^{n+1}$ is an isomorphism onto since $f^{n+1}$ is a homotopy equivalence. Also

$$
i_{n}: \pi_{n}\left(K_{n+1}\right) \rightarrow \pi_{n}(K), \quad i_{n}: \pi_{n}\left(L_{n+1}\right) \rightarrow \pi_{n}(L)
$$

are isomorphisms onto and obviously $f_{n} i_{n}=i_{n} f_{n}^{n+1}$. Therefore $f_{n}$ is an isomorphism onto, for each $n=1,2, \cdots$, and the lemma follows from Theorem 1.

We are now ready to prove Theorem 13 . This will obviously follow when we have proved it for a countable CW-complex, $K$, which is connected if $p=\operatorname{dim} K>0$. We first assume $K$ to be finite and use induction on the number of cells in $K$, starting with the trivial case $K=K^{0}$. Let $p>0$ and let $K_{0}=K-e^{p}$, where $e^{p}$ is any $p$-cell in $K$. Assume that there is a finite complex, $L_{0}$, with a simplicial subdivision $L_{0}^{\prime}$, which is related to $K_{0}$ by a homotopy equivalence $f_{0}: K_{0} \rightarrow L_{0}$. Let $e^{p}$ be attached to $K_{0}$ by a map $g: \partial E^{p} \rightarrow K_{0}$, where $e^{p}=E^{p}-\partial E^{p}$. Assuming that $L_{0} \cap E^{p}=0$ let $e^{p}$ be attached to $L_{0}$ by a map

$$
h_{0} \simeq f_{0} g: \partial E^{p} \rightarrow L_{0},
$$

which is simplicial with respect to $L_{0}^{\prime}$ and some triangulation of $\partial E^{p}$. The result is a complex, $L$, which has a simplicial subdivision, according to Lemma 2. The theorem, for $K$ finite, now follows from the induction and Lemma 3.

Now let $K$ be infinite. In order to repeat the above construction an infinite number of times we shall, so to speak, "spread $L_{0}$ out" so that $L$ may be locally finite. Assume that there is a sequence of locally finite complexes, $L_{0} \subset L_{1} \subset \cdots$, such that $L_{n}$ is connected if $n>0$, and a sequence of subcomplexes, $P_{n} \subset L_{n}$, such that:

(9.1) (a) $L_{n-1}-P_{n-1}=U_{n-1}$ is open in $L_{n}$ and $L_{n-1} \subset U_{n}\left(L_{-1}=P_{-1}\right.$ $=0$ ), 
(b) $P_{n}$ is a deformation retract of $L_{n}$,

(c) if $n>m>1$ the injection homomorphism $\pi_{m-1}\left(L_{m}\right) \rightarrow \pi_{m-1}\left(L_{n}\right)$ is an isomorphism onto,

(d) there is a sequence of homotopy equivalences, $f^{n}:\left(K^{n}, K^{n-1}\right)$ $\rightarrow\left(L_{n}, L_{n-1}\right)$, such that $f^{n} \mid K^{n-1}=f^{n-1}$,

(e) $L_{n}$ has a simplicial subdivision, $L_{n}^{\prime}$, of which $L_{n-1}^{\prime}$ is a subcomplex.

(f) $\operatorname{dim} L_{n}=\operatorname{dim} K^{n} \leqq n$.

Then we define the topological space

$$
L=\bigcup_{n=0}^{p} L_{n} \quad(p=\operatorname{dim} K \leqq \infty),
$$

in which a subset $X \subset L$ is closed (open) if, and only if, $X \cap L_{n}$ is a closed (relatively open) subset of $L_{n}$, for each $n \geqq 0$. Clearly $L_{n}$ keeps its own topology in $L$ and since each $L_{n}$ is a locally finite complex it follows that $L$ is a CW-complex, which consists of the cells in $L_{0}, L_{1}, \cdots$. I say that $L$ is locally finite. For $U_{n-1} \subset L_{n-1} \subset U_{n}$ and $U_{n-1}$ is open in $L_{n}$ and hence in $U_{n}$. Therefore, given $m \geqq 0$, it follows by induction on $n$ that $U_{m}$ is open in $U_{n}$ and hence in $L_{n}$ for every $n>m$. Therefore $U_{m}$ is open in $L$. Since $L_{n-1} \subset U_{n}$ it follows that any point $q \in L$ is in $U_{n}$ for some $n$. Let $q \in U_{n}$ and let $Q \subset L_{n}$ be a finite subcomplex, of which $q$ is an inner point, according to the topology of $L_{n}$. Since $U_{n}$ is open in $L$ it follows that $q$ is an inner point of $Q$, according to the topology of $L$. Therefore $L$ is locally finite.

Since $L$ is locally finite it follows from (D), in $\$ 5$ above, that any compact subset is contained in a finite subcomplex of $L$. It is therefore contained in $L_{n}$, for some $n \geqq 0$, and it follows without difficulty from (9.1c) that the injection homomorphism $\pi_{m-1}\left(L_{m}\right) \rightarrow \pi_{m-1}(L)$ $(m>1)$ is an isomorphism onto. Therefore it follows from (9.1d) and Lemma 4 that $K \equiv L$. Clearly

$$
L^{\prime}=\bigcup_{n} L_{n}^{\prime}
$$

is a (locally finite) simplicial subdivision of $L$. Finally $\operatorname{dim} L$ $=p=\operatorname{dim} K$ and the theorem follows.

Now assume that $L_{n}, P_{n}$ have been constructed for $n=1, \cdots, r \geqq 0$ so as to satisfy (9.1) with $n \leqq r$. If $K^{r+1}=K^{r}$ we define $L_{r+1}=L_{r}$, $P_{r+1}=P_{r}$. Let $K^{r+1} \neq K^{r}$ and let $X_{a}$ be the complex covering the half line $\langle a, \infty)$, which has 0 -cells at the points $a+i(i=0,1, \cdots)$. Assuming that the topological product $P_{r} \times X_{0}$ has no point in common with $L_{r}$, we identify each point $x \in P_{r}$ with $(x, 0) \in P_{r} \times 0$ so as to form a locally finite complex

$$
M_{r}=L_{r} \cup\left(P_{r} \times X_{0}\right) .
$$


This has the simplicial subdivision $L_{r}^{\prime} \cup\left(P_{r} \times X_{0}\right)^{\prime}$, where $\left(P_{r} \times X_{0}\right)^{\prime}$ is formed by starring the prisms $\sigma \times\langle i, i+1\rangle(i=0,1, \ldots)$, for each simplex, $\sigma$, in the subcomplex of $L_{r}^{\prime}$ which covers $P_{r}$. Clearly $L_{r}$ is a deformation retract of $M_{r}$, whence $g^{r}: K^{r} \rightarrow M_{r}$, given by $g^{r} x=f^{r} x$ $\left(x \in K^{r}\right)$, is a homotopy equivalence. It obviously follows from (9.1b) that $P_{r} \times X_{0}$ is a deformation retract of $M_{r}$ and hence that $P_{r} \times k$ is a deformation retract of $M_{r}$, for each $k=1,2, \cdots$. Therefore any map $\partial E^{r+1} \rightarrow M_{r}$ is homotopic to a map in $P_{r} \times k$. We now repeat the construction used in the finite case, attaching an $(r+1)$-cell, $e_{\mathbf{k}}^{r+1}$, to $M_{r}$ by a suitable map $\partial E_{\mathbf{k}}^{r+1} \rightarrow P_{r} \times k(k>0)$, where $e_{1}^{r+1}, e_{2}^{r+1}, \cdots$ are the $(r+1)$-cells in $K$. The result is a locally finite complex, $L_{r+1}$, which satisfies $(9.1 \mathrm{~d}, \mathrm{e}, \mathrm{f})$ with $n \leqq r+1$. We define

$$
P_{r+1}=\left(P_{r} \times X_{1}\right) \cup\left\{e_{k}^{r+1}\right\} .
$$

Clearly $P_{r} \times X_{1}$ is a deformation retract of $P_{r} \times X_{0}$, and hence of $M_{r}$. Therefore $P_{r+1}$ is a deformation retract of $L_{r+1}$. Therefore (9.1b), and obviously (9.1a), is satisfied with $n \leqq r+1$. Since $L_{r}$ is a deformation retract of $M_{r}$ and $L_{r+1}$ is formed by attaching $(r+1)$-cells to $M_{r}$ it follows that (9.1c), and hence all the conditions (9.1), is satisfied with $n \leqq r+1$. Starting with $L_{0}=P_{0}=K^{0}$ the theorem now follows by induction on $r$.

10. Proof of Lemma 2. An $n$-element, $E^{n}$, in an $n$-sphere, $S^{n}$, will be called a hemisphere if, and only if, the closure of $S^{n}-E^{n}$ is also an $n$-element. Let $f: \sigma^{n} \rightarrow \sigma^{k}$ be a barycentric map of an $n$-simplex, $\sigma^{n}$, onto a $k$-simplex $\sigma^{k}(k \leqq n)$. Assuming that $\sigma^{k}$ does not meet $\sigma^{n}$ let $\Gamma$ be the mapping cylinder of $f$. We shall prove:

Lemma $5 . \Gamma$ is an $(n+1)$-element and $\sigma^{n}$ is a hemisphere in $\partial \Gamma$.

Before proving this we deduce a corollary and show that it implies Lemma 2. Let $\sigma_{1}^{n}$ be a face of an $(n+1)$-simplex, $\sigma^{n+1}$. Let $f_{1}: \sigma_{1}^{n} \rightarrow \sigma^{k}$ be a barycentric map of $\sigma_{1}^{n}$ onto $\sigma^{k}$ and, assuming that $\sigma^{k}$ does not meet $\sigma_{1}^{n+1}$, let $E^{n+1}$ be the space formed by identifying each point $p \in \sigma_{1}^{n}$ with $f_{1} p$. Obviously $E^{n+1}$ is homeomorphic to $\sigma_{0}^{n+1} \cup \Gamma$, where $\Gamma$ means the same as in Lemma 5 and $\sigma_{0}^{n+1}$ is an $(n+1)$-simplex, of which $\sigma^{n}=\sigma_{0}^{n+1} \cap \Gamma$ is a face. Since $\sigma^{n}$ is a hemisphere both of $\partial \sigma_{0}^{n+1}$ and of $\partial \Gamma$ we obviously have the corollary:

Corollary. $E^{n+1}$ is an $(n+1)$-element.

Let an $n$-cell, $e^{n}$, be attached to a complex, $K$, by means of a map, $\partial E^{n} \rightarrow K$, which is simplicial with respect to a simplicial subdivision, $K^{\prime}$, of $K$ and some triangulation, $S$, of $\partial E^{n}$. Let $p_{0}$ be an interior 
point of $E^{n}$. When $e^{n}$ is attached to $K$ each simplex $\sigma^{r+1}=p_{0} \sigma^{r}$, where $\sigma^{r}$ is a simplex of $S$, is transformed into an $(r+1)$-element $E^{r+1}$, according to the above corollary. We triangulate $K^{\prime} \cup e^{n}$ by starring all these $(r+1)$-elements, in order of increasing dimensionality. The result is a simplicial subdivision of $K \cup e^{n}$, which contains $K^{\prime}$ as a subcomplex. Lemma 2 follows from this construction, carried out in each of the cells $e_{i}^{n}$.

We now prove Lemma 5 , using a method suggested by M. H. A. Newman. Let $\sigma^{n}$ be a rectilinear simplex in Euclidean space $R^{n}$. Let $b_{0}, \cdots, b_{k}$ be the vertices of $\sigma^{k}$ and let $\sigma_{i}$ be the face of $\sigma^{n}$, whose vertices are those which are mapped by $f$ on $b_{i}$. Let $a_{i}$ be the centroid of $\sigma_{i}$, let $\sigma_{0}^{k}$ be the $k$-simplex $a_{0} \cdots a_{k}$ and let $g: \sigma^{n} \rightarrow \sigma_{0}^{k}$ be the barycentric map, which is determined by $g \sigma_{i}=a_{i}$. Then $g a_{i}=a_{i}$, whence $g g x=g x$ for each $x \in \sigma^{n}$, or $g^{2}=g$. Also $c \in \sigma_{0}^{k}$, where $c$ is the centroid of $\sigma^{n}$, since $a_{i}$ is the centroid of $\sigma_{i}$, and it follows from the definition of a barycentric map that $g c=c$.

We treat $R^{n}$ as a vector space, taking $c$ to be the origin or null vector, which we also denote by 0 . We shall use small Roman letters to denote vectors in $R^{n}$ and small Greek letters, as in $\lambda x+\mu y$, to denote (real) scalar multipliers. Let $\theta_{\lambda}: \sigma^{n} \rightarrow \sigma^{n}$ be the map which is given by

$$
\theta_{\lambda} x=(1-\lambda) x+\lambda g x / 2
$$$$
\left(0 \leqq \lambda \leqq 1 ; x \in \sigma^{n}\right) .
$$

I say that if $\lambda<1$ the map $\theta_{\lambda}$ is nonsingular. For $\theta_{\lambda}$ is a linear map, such that $\theta_{\lambda} 0=0$, and if it is singular, then $\theta_{\lambda} x=0$ for some nonzero vector $x \in \sigma^{n}$. Since $g^{2}=g$ it follows from the linearity of $g$ that

$$
g \theta_{\lambda} x=(1-\lambda) g x+\lambda g x / 2=(1-\lambda / 2) g x .
$$

Therefore $\theta_{\lambda} x=0$ implies, first, $g x=0$, and then $(1-\lambda) x=0$, or $x=0$ if $\lambda<1$, which proves the assertion.

I say that, if $0 \leqq \lambda<\mu \leqq 1$, then $\theta_{\mu} \sigma^{n}$ is in the interior of $\theta_{\lambda} \sigma^{n}$. For $\theta_{\lambda} x$ is the point which divides the segment joining $x$ to $g x / 2$ in the ratio $\lambda:(1-\lambda)$. When we have shown that the simplex $\theta_{1} \sigma_{0}^{k}$ is in the interior of $\theta_{\lambda} \sigma^{n}(\lambda<1)$ it will follow that the vertices of $\theta_{\mu} \sigma^{n}(\mu>\lambda)$ lie on rectilinear segments, which join the vertices of $\theta_{\lambda} \sigma^{n}$ to interior points of $\theta_{\lambda} \sigma^{n}$. Hence, and since $\mu>\lambda$, they are interior points of $\theta_{\lambda} \sigma^{n}$. Since $\theta_{\rho}$ is a linear map the assertion will follow. Thus we have to prove that $\theta_{1} \sigma_{0}^{k}$ is inside $\theta_{\lambda} \sigma^{n}$, or that any point $g a / 2\left(a \in \sigma^{n}\right)$ is of the form $\theta_{\lambda} x$, for some interior point $x \in \sigma^{n}$. Let $\rho=1 /(2-\lambda)$. Then

$$
\begin{aligned}
\theta_{\lambda} \rho g a & =(1-\lambda) \rho g a+\lambda \rho g^{2} a / 2 \\
& =\rho(1-\lambda / 2) g a=g a / 2 .
\end{aligned}
$$


But $\rho<1$ since $\lambda<1$. Therefore $\rho g a$ is an interior point of $\sigma^{n}$ and the assertion is proved.

The set of values $\lambda \in I$, such that a given point, $x \in \sigma^{n}$, does not lie in $\theta_{\lambda} \sigma^{n}$, is obviously open. Therefore there is a maximum, $\phi(x)$, of the values of $\lambda$ such that $x \in \theta_{\lambda} \sigma^{n}$. It follows without difficulty from the preceeding paragraph that $\phi$ is a continuous, real-valued function of $x$, such that
(a) $\phi(x)>0$
(b) $\phi(x)=0$
(c) $x \in \theta_{\lambda} \sigma^{n}$

if $x \in \sigma^{n}-\partial \sigma^{n}$,

if $x \in \partial \sigma^{n}$,

if, and only if, $\lambda \leqq \phi(x)$.

We now imbed $\sigma^{n}$ in $\sigma^{n} \times I$, in such a way that $x=(x, 0)$ if $x \in \sigma^{n}$, and take $\sigma^{k}$ to be the simplex $\theta_{1} \sigma_{0}^{k} \times 1$ and $f: \sigma^{n} \rightarrow \sigma^{k}$ to be the map given by

$$
f x=\left(\theta_{1} x, 1\right)=(g x / 2,1) .
$$

If $x$ is fixed and $\lambda$ varies from 0 to 1 we may regard the locus of the point $\left(\theta_{\lambda} x, \lambda\right)$ as the linear segment in $\sigma^{n} \times I$, which joins $x \in \sigma^{n}$ to $f x \in \sigma^{k}$. If $\theta_{\lambda} x=\theta_{\lambda} y$ it follows from (10.2) that $g x=g y$ and from (10.1) that $x=y$ if $\lambda<1$. Therefore no two of these linear segments have an inner point in common and we may take $\Gamma$ to be the union of the sets $\theta_{\lambda} \sigma^{n} \times \lambda$ for each $\lambda \in I$. It follows from (10.3c) that $\Gamma$ is the set of all points $(x, \lambda)$ such that $0 \leqq \lambda \leqq \phi(x)$.

Let $\psi: \sigma^{n} \rightarrow I$ be the real-valued function which is defined by $\psi(\rho v)=1-\rho$ for each vector $v \in \partial \sigma^{n}$ and each $\rho \in I$. Then the subset of $\sigma^{n} \times I$, which consists of all points $(x, \lambda)$ such that $0 \leqq \lambda \leqq \psi(x)$, is the join, $\sigma^{n+1}$, of $\sigma^{n}$ and the point $(0,1)$. A homeomorphism (onto), $h: \Gamma \rightarrow \sigma^{n+1}$, is defined by

$$
h(x, \lambda)=\{x, \rho(x) \lambda\},
$$

where $0 \leqq \lambda \leqq \phi(x)$ and $\rho(x)=\psi(x) / \phi(x)$ if $x \in \sigma^{n}-\partial \sigma^{n}, \rho(x)=0$ if $x \in \partial \sigma^{n}$. Clearly $h \sigma^{n}=\sigma^{n}$ and $\sigma^{n}$ is a hemisphere of $\partial \sigma^{n+1}$. Therefore Lemma 5 is established.

Let $K, L$ be simplicial complexes and let $f: K \rightarrow L$ be a simplicial map. Let $C$ be the "combinatorial" mapping cylinder of $f$, defined as in [1], and $\Gamma$ the "topological" mapping cylinder defined as in $\$ 8$ above. Let $\sigma$ be any simplex in $K$ and let $C_{\sigma} \subset C, \Gamma_{\sigma} \subset \Gamma$ be the mapping cylinders of the map, $\sigma \rightarrow f \sigma$, which is determined by $f$. It follows from Lemma 5 that $\Gamma$ is a polyhedral complex, whose (closed) cells are the simplexes in $K$ and in $L$ and the elements $\Gamma_{\sigma}$, for each simplex, $\sigma$, in $K$. Since $\Gamma_{\sigma}$ is an element it may be starred from an internal 
point. Since $C_{\sigma}$ is also a star an easy induction shows that $C$ is combinatorially isomorphic to the triangulation of $\Gamma$, which is obtained by starring the elements $\Gamma_{\sigma}$ in order of increasing dimensionality. Hence the conjecture in the footnote on p. 1235 of [3] is easily verified.

\section{REFERENCES}

1. J. H. C. Whitehead, Proc. London Math. Soc. vol. 45 (1939) pp. 243-327.

2. - Ann. of Math. vol. 42 (1941) pp. 409-428.

3. —- Ann. of Math. vol. 42 (1941) pp. 1197-1239.

4. - - Proc. London Math. Soc. vol. 48 (1945) pp. 243-291.

5. - On simply connected, 4-dimensional polyhedra, Comment. Math. Helv. vol. 22 (1949) pp. 48-92.

6. - On the homotopy type of ANR's, Bull. Amer. Math. Soc. vol. 54 (1948) pp. 1133-1145.

7. - Note on a theorem due to Borsuk, Bull. Amer. Math. Soc. vol. 54 (1948) pp. 1125-1132.

8. K. Reidemeister, Topologie der Polyeder, Leipzig, 1938.

9. R. H. Fox, Ann. of Math. vol. 42 (1941) pp. 333-370.

10. —- Ann. of Math. vol. 44 (1943) pp. 40-50.

11. N. E. Steenrod, Ann. of Math. vol. 48 (1947) pp. 290-320.

12. S. Eilenberg, Fund. Math. vol. 32 (1939) pp. 167-175.

13. - Trans. Amer. Math. Soc. vol. 61 (1947) pp. 378-417.

14. S. Eilenberg and N. E. Steenrod, Foundations of Algebraic topology, not yet published.

15. W. Hurewicz, K. Akademie van Wetenschappen, Amsterdam, Proceedings vol. 38 (1935) pp. 521-528.

16. - K. Akademie van Wetenschappen, Amsterdam, Proceedings vol. 39 (1936) pp. 215-224.

17. H. Hopf, Comment. Math. Helv. vol. 14 (1942) pp. 257-309.

18. - Comment. Math. Helv. vol. 15 (1942) pp. 27-32.

19. - Comment. Math. Helv. vol. 17 (1945) pp. 307-326.

20. Claude Chevalley, Theory of Lie groups, Princeton, 1946.

21. Charles Ehresmann, Bull. Soc. Math. France, vol. 72 (1944) pp. 27-54.

22. W. Hurewicz and N. E. Steenrod, Proc. Nat. Acad. Sci. U.S.A. vol. 27 (1941) pp. 60-64.

23. P. Alexandroff and H. Hopf, Topologie, Berlin, 1935; Also Ann Arbor, 1945.

24. N. Bourbaki, Topologie gênérale, vol. 3, Paris, 1940.

25. A. L. Blakers, Ann. of Math. vol. 49 (1948) pp. 428-461.

Magdalen College, Oxford University 